\title{
H. Suura*
}

School of Physics and Astronomy, University of Minnesota

Minneapolis, Minnesota 55455

and

Bing-Lin Young

Ames Laboratory-USAEC and Department of Physics

lowa State University, Ames, lowa 50010

\section{ABSTRACT}

Functional integration method is applied to derive Ward-

Takahashi identities involving the energy-momentum tensor, currents, and fields. In the process of the derivation, we have introduced a variational method to derive conservation laws, in which the improved energy-momentum tensor of Callen-Coleman-Jackiw is obtained. Establishing the correspondence between the c-number relations obtained in the functional integration method and the q-number ones resulting from the conventional way, we demonstrated that the two approaches of the quantum.field-theory give identical results for the perturbation expansion, canonical equal-time commutation relations, etc. They are, therefore, equivalent.

\footnotetext{
"Research support in part by the Atomic Energy Commission.
} 


\section{DISCLAIMER}

This report was prepared as an account of work sponsored by an agency of the United States Government. Neither the United States Government nor any agency Thereof, nor any of their employees, makes any warranty, express or implied, or assumes any legal liability or responsibility for the accuracy, completeness, or usefulness of any information, apparatus, product, or process disclosed, or represents that its use would not infringe privately owned rights. Reference herein to any specific commercial product, process, or service by trade name, trademark, manufacturer, or otherwise does not necessarily constitute or imply its endorsement, recommendation, or favoring by the United States Government or any agency thereof. The views and opinions of authors expressed herein do not necessarily state or reflect those of the United States Government or any agency thereof. 


\section{DISCLAIMER}

Portions of this document may be illegible in electronic image products. Images are produced from the best available original document. 


\section{INTRODUCTION}

In 1948 Feynman' proposed a path integral formulation of quantum mechanics which was later extended to the treatment of the relativistic quantum field-theory, praticularly, the quantum electrodynamics. 2 Over the last one and a half decades, many authors have investigated this functional integration formulation of quantum field-theory and demonstrated in the few cases investigated, that this formulation reproduces results of the perturbation theory. 3,4 However, the hope that this method would offer a closed solution of the quantum field-theory to bypass the difficulties of the perturbation calculation has not been real ized.

The formulation has not been particularly popular until now due to its unfamiliarity and mathematical difficulties that have not been completely worked out. Irrespective of its weakness in performing actual calculations, the functional integration method has recently attracted wide attention. It has been used to construct renormalizable gauge field-theory ${ }^{5}$ and representations of dual resonance amplitudes. 6 .

In this article, we shall discuss an application of the functional integration formulation to derive general Ward-Takahashi identities (called W-T identities hereafter) involving the energy-momentum tensor, its trace, and gauge currents. The conventional method in deriving W-T identities, involving the use of equal-time commutators among field operators, is not suitable for the present purpose, since the energymomentum tensor contains time derivatives of field operators. Another interesting method, due to Gross and Jackiw, ${ }^{7}$ using the idea of the 
covariant T-product, has been successfully applied in a few simple cases. $^{8}$ The method, however, seems to be quite involved in more complicated cases. The functional integration formulation appears to be a more economical machinery to achieve the purpose.

In the functional integration approach, like the conventional method, one starts with a definite Lagrangian to provide a dynamical framework; nevertheless, unlike the conventional approach, equations of motion of the fields will not enter the calculation, final results satisfying automatically constraints of the equations of motion.

In Sec. II, we obtain local transformations of fields which generate, in a variational method, conservation laws when applied to a Lagrangian. Especially, we derive gauge currents, the improved energy-momentum tensor of Callen, Coleman, and Jackiw, ${ }^{9}$ and its trace. The results of the gauge currents agree with those of Gell-Mann and Lévy. ${ }^{10}$ These same local transformations are used in Sec. III and IV as changes of functional integration variables in the path integral representations of $\mathrm{N}$-point functions ( $n-p f ! s)$, thereby producing the W-T identities. The functional formulation will be reviewed briefly in Sec. 111 , where the procedure of deriving W-T identities will also be illustrated. In Sec. IV, W-T identities and trace identities of n-pf's involving the energy-momentum tensor, vector currents, and axial-vector currents are derived. In Sec. $V$, we discuss the general relations between $W-T$ identities, conservation laws, and equal-time commutators. Section VI is reserved for concluding remarks.

In Appendix A, we derive two expressions of the generating functional for a vector gluon interaction. The Jacobians of the transformations 
of fields will be discussed in Appendix B. The Feynman rules for the energy-momentum tensor will be given in Appendix $C$.

\section{GENERAL CONSERVATION LAWS}

Assume that a system consisting of fields $\varphi_{\alpha}, \alpha=1,2, \cdots n$, is described by a Lagrangian

$$
\mathfrak{L}\left(\varphi_{\alpha}, \varphi_{\alpha, \mu}\right)
$$

where

$$
\varphi_{\alpha, \mu} \equiv \frac{\partial}{\partial x^{\mu}} \varphi_{\alpha}(x)
$$

The action integral is defined as usual by

$$
I \equiv \int \mathcal{L}\left(\varphi_{\alpha}, \varphi_{\alpha, \mu}\right) d^{4} x
$$

We consider a variation of the fields

$$
\varphi_{\alpha}(x) \rightarrow \varphi_{\alpha}(x)+\delta \varphi_{\alpha}(x)
$$

where

$$
\delta \varphi_{\alpha}(\dot{x})=\omega_{[\nu]}(x) F_{\alpha}^{[\nu]}\left(\varphi_{\beta}, \varphi_{\beta, \lambda}\right)+\partial_{\mu}{ }^{\omega}[\nu](x) G_{\alpha}{ }^{\mu[\nu]}\left(\varphi_{\beta}, \varphi_{\beta, \lambda}\right)
$$

$[\mu]$ denotes a set of indices $\mu_{1}, \mu_{2}, \cdots \mu_{m} ; \omega_{[\nu]}(x)$ is an infinitesimal tensor function vanishing on the 4-dimensional (infinite) boundary surface together with its first m-th derivatives. In the following consideration we need $m=2$. We also have from Eq. (2.3), 


$$
\begin{aligned}
\delta \varphi_{\alpha, \rho}(x) & =\omega_{[\nu]}(x) \partial_{\rho} F_{\alpha}^{[\cdot \nu]}\left(\varphi_{\beta}, \varphi_{\beta, \lambda}\right)+\partial_{\rho}^{\omega}[\nu] \\
& +\partial_{\mu} \omega_{[\nu]}(x) \partial_{\rho} \sigma_{\alpha} G_{\alpha}^{\mu[\nu]}\left(\varphi_{\beta}, \varphi_{\beta, \lambda}\right)+\partial_{\rho} \partial_{\mu, \lambda}{ }^{\omega}{ }^{\omega}[\nu] \\
& (x) G_{\alpha}^{\mu[\nu]}\left(\varphi_{\beta}, \varphi_{\beta, \lambda}\right)
\end{aligned}
$$

The corresponding variation of the action integral is

$$
\begin{aligned}
\delta l & =\int d^{4} \times \omega[\mu]\left\{\frac{\partial \mathscr{L}}{\partial \varphi_{\alpha}} F_{\alpha}^{[\mu]}+\frac{\partial \mathcal{L}}{\partial \varphi_{\alpha, \lambda}} \partial_{\lambda} F_{\alpha}^{[\mu]}-\partial_{\lambda}\left(\frac{\partial \mathcal{L}}{\partial \varphi_{\alpha, \lambda}} F_{\alpha}^{[\mu]}\right)\right. \\
& \left.-\partial_{\lambda}\left(\frac{\partial \mathcal{L}}{\partial \varphi_{\alpha}} G_{\alpha}^{\lambda[\mu]}+\frac{\partial \mathcal{L}}{\partial \varphi_{\alpha, \nu}} \partial_{\nu} G_{\alpha}^{\lambda[\mu]}\right)+\partial_{\lambda} \partial_{\rho}\left(\frac{\partial \mathcal{L}}{\partial \varphi_{\alpha, \rho}} G_{\alpha}^{\lambda[\mu]}\right)\right\}
\end{aligned}
$$

To show that Eqs. (2.2) and (2.3) will lead to a conservation law, let us rewrite Eq. (2.5)

$$
\delta I=\int d^{4} x \omega_{[\mu]}(x)\left\{[\mathscr{D}] \varphi_{\alpha} F_{\alpha}^{[\mu]}+\partial_{\lambda}\left([\Sigma] \varphi_{\alpha} G_{\alpha}^{\lambda[\mu]}\right)\right\}
$$

where

$$
[£] \varphi_{\alpha} \equiv \frac{\partial \mathscr{L}}{\partial \varphi_{\alpha}}-\partial_{\rho} \frac{\partial \mathcal{L}}{\partial \varphi_{\alpha, \rho}}
$$

is the Euler-Lagrange equation for $\varphi_{\alpha}$. Upon the use of the equations of motion of $\varphi_{\alpha}$, $\delta 1$ vanishes. Hence the terms in the curly bracket of (2.5) must lead to a conservation or partial conservation law.

In obtaining the above expression, partial integrations have been freely used. The quantity in the curly bracket of $(2.5)$ corresponds to the divergence of a current density arising from the transformation (2.3). In the following, we list various conservation laws which can be obtained from (2.5). Let us emphasize that the space-time coordinates are not transformed in the present derivation of conservation laws. 
A. The Euler-Lagrangian Equation

Take

$$
\begin{aligned}
& F_{\alpha}^{[\mu]}=\delta_{\alpha \beta} \quad(\beta \text { a fixed index }) \\
& G_{\alpha}^{\lambda[\mu]}=0
\end{aligned}
$$

then

$$
\delta 1=\int d^{4} x \omega(x)\left\{\frac{\partial \mathcal{L}}{\partial \varphi_{\alpha}}-\partial_{\lambda}\left(\frac{\partial \mathcal{L}}{\partial \varphi_{\alpha, \lambda}}\right)\right\}
$$

B. Canonical Energy-Momentum Tensor

$$
\begin{aligned}
F_{\alpha}^{[\mu]} & =\partial^{\mu} \varphi_{\alpha} \\
G_{\alpha}^{\lambda[\mu]} & =0 \\
& \therefore I \\
\delta ! & -\int d^{4} x \omega^{\nu}(x) \partial^{\lambda} T_{\lambda \nu}
\end{aligned}
$$

where

$$
T_{\lambda \nu}=\frac{\partial \mathcal{L}}{\partial \varphi_{\alpha,} \lambda} \varphi_{\alpha, \nu}-g_{\lambda \nu}{ }^{\mathcal{L}}
$$

is the canonical energy-momentum tensor. Notice that

$$
\delta \varphi_{\alpha}=\omega_{\mu}(x) \partial^{\mu} \varphi_{\alpha}(x)
$$

is actually the local variation of $\varphi_{\alpha}$ due to a coordinate dependent translation. 11 


\section{Angular Momentum Tensor}

$$
\begin{aligned}
F_{\alpha}^{\mu \nu} & =x^{\mu} \partial^{\nu} \varphi_{\alpha}-x^{\nu} \partial^{\mu} \varphi_{\alpha}+\left(\Sigma^{\mu \nu}\right)_{\alpha \beta} \varphi_{\beta} \\
G_{\alpha}^{[\mu]} & =0
\end{aligned}
$$

where the spin tensor, $\Sigma_{\mu \nu}$, is given by .

$$
\left(\Sigma_{\mu \nu}\right)_{\alpha \beta}=\left\{\begin{array}{c}
0 \text { for scalar and pseudoscalar field } \\
\frac{1}{4}\left[\gamma_{\mu}, \gamma_{\nu}\right]_{\alpha \beta} \text { for spin } \frac{1}{2} \text { field } \\
g_{\mu \alpha} g_{\nu \beta}-g_{\mu \beta} g_{\nu \alpha} \text { for spin } 1 \text { field. }
\end{array}\right.
$$

We obtain

$$
\delta 1=\int d^{4} x \omega^{\mu \nu}(x)\left\{\partial^{\lambda} M_{\lambda \mu \nu}+\Delta_{\mu \nu}\right\},
$$

where

$$
\begin{aligned}
M_{\lambda \mu \nu} & =T_{\lambda \mu}{ }{ }-T_{\lambda \nu}{ }^{x_{\mu}}-\frac{\partial \mathcal{L}}{\partial \varphi_{\alpha,} \lambda}\left(\Sigma_{\mu \nu}\right)_{\alpha \beta} \varphi_{\beta} \\
\Delta_{\mu \nu} & =\frac{\partial \mathcal{L}}{\partial \varphi_{\alpha,}{ }^{\mu}} \varphi_{\alpha, \nu}-\frac{\partial \mathcal{L}}{\partial \varphi_{\alpha}, \nu} \varphi_{\alpha, \mu}+\frac{\partial \mathcal{L}}{\partial \varphi_{\alpha}}\left(\Sigma_{\mu \nu}\right)_{\alpha \beta} \varphi_{\beta}+\frac{\partial \mathcal{L}}{\partial \varphi_{\alpha, \lambda}}\left(\Sigma_{\mu \nu}\right)_{\alpha \beta} \varphi_{\beta, \lambda}
\end{aligned}
$$

The Lorentz covariance requires as an identity ${ }^{12}$

$$
\Delta_{\mu \nu} \equiv 0,
$$

so that we recover the conservation of angular momentum from Eq. (2.12). 
Here again, we see that Eq. $(2.10)$ is the local variation of the fields due to a coordinate dependent homogeneous Lorentz transformation.

\section{Symmetric Energy-Momentum Tensor}

In the conventional approach, the symmetric energy-momentum tensor can not be derived from a space-time variation of the fields; it is defined in terms of the Belinfante tensor, 13

$$
\theta_{\mu \nu}^{B}=T_{\mu \nu}+\frac{1}{2} \partial^{\lambda}\left[\frac{\partial \mathcal{L}}{\partial \varphi_{\alpha},}\left(\Sigma_{\mu \nu}\right)_{\alpha \beta} \dot{\varphi}_{\beta}+\frac{\partial \mathcal{L}}{\partial \varphi_{\alpha}{ }^{\mu}}\left(\Sigma_{\nu \lambda}\right)_{\alpha \beta} \varphi_{\beta}+\frac{\partial \mathcal{L}}{\partial \varphi_{\alpha}{ }_{\nu}}\left(\Sigma_{\mu \lambda}\right)_{\alpha \beta} \varphi_{\beta}\right]
$$

In order to prove that $\theta_{\mu \nu}^{B}$ is symmetric in $\mu$ and $\nu$, equations of motion have to be used since

$$
\theta_{\mu \nu}^{B}-\theta_{\nu \mu}^{B}=-\left[\frac{\partial \mathcal{L}}{\partial \varphi_{\alpha}}-\partial_{\lambda} \frac{\partial \mathcal{L}}{\partial \varphi_{\alpha, \lambda}}\right]\left(\Sigma_{\mu \nu}\right)_{\alpha \beta} \varphi_{\beta}
$$

after identity (2.14) is used. Indeed, in spinor electrodynamics, for instance, the expression of $\theta_{\mu \nu}^{B}$ calculated from Eq. (2.15) is not symmetric in $\mu$ and $\nu$, unless equations of motion are used to rearrange some of the terms. To make $\theta_{\mu \nu}^{B}$ explicitly symmetric, we define

$$
\bar{\theta}_{\mu \nu} \equiv \frac{1}{2}\left(\theta_{\mu \nu}^{B}+\theta_{\nu \mu}^{B}\right)=\theta_{\mu \nu}^{B}+\frac{1}{2}\left[\frac{\partial \mathcal{L}}{\partial \varphi_{\alpha}}-\partial_{\lambda} \frac{\partial \mathcal{L}}{\partial \varphi_{\alpha, \lambda}}\right]\left(\Sigma_{\mu \nu}\right)_{\alpha \beta} \varphi_{\beta}
$$

which is, needless to say, equal to $\theta_{\mu \nu}^{B}$. We shall see that our variational method generates the tensor $\bar{\theta}_{\mu \nu}$. Consider the field variations

$$
\begin{gathered}
F_{\alpha}^{[\mu]}=\varphi_{\alpha}^{\mu}, \\
G_{\alpha}^{\lambda[\mu]}=\frac{1}{2}\left(\Sigma^{\lambda \mu}\right)_{\alpha \beta} \varphi_{\beta} .
\end{gathered}
$$


It is easy to check that the corresponding variation of the action integral is

$$
\delta 1=-\int d^{4} x \omega^{\mu}(x) \partial^{\lambda} \bar{\theta}_{\lambda \mu} .
$$

The variations of fields, Eq. (2.18), do not arise from a known spacetime transformation. Still Eq. (2.19) does not determine the energymomentum tensor uniquely, it admits further modification by divergentless symmetric tensors which do not contribute to the energy-momentum operator. Therefore, we shall define our final form of energy momentum tensor to be

$$
\theta_{\lambda \mu}=\bar{\theta}_{\lambda \mu}+x_{\lambda \mu}
$$

where $x_{\lambda \mu}$, to be determined from the consideration of the trace of $\theta_{\lambda \mu}$, satisfies the constraints:

$$
x_{\lambda \mu}=x_{\mu \lambda}, \quad \partial^{\lambda} x_{\lambda \mu}=0
$$

and

$$
\int x_{o \mu} d^{3} x=0
$$

The last condition is needed to make sure that the energy-momentum vector is still

$$
P_{\mu}=\int \theta_{o \mu} d^{3} x=\int T_{o \mu} \cdot d^{3} x
$$




\section{E. Trace of the Improved Energy-Momentum Tensor}

$$
\begin{aligned}
& \text { We take } \\
& \mathrm{F}_{\alpha}^{[\mu]}=\mathrm{d}_{\alpha} \varphi_{\alpha} \\
& \mathrm{G}_{\alpha}^{\lambda[\mu]}=0
\end{aligned}
$$

where $d_{\alpha}$ is the canonical dimension of $\varphi_{\alpha}$,

$$
d_{\alpha}= \begin{cases}1 & \text { for bosons } \\ \frac{3}{2} & \text { for fermions }\end{cases}
$$

The variation (2.22) gives rise to the following change in the action

$$
\delta 1=-\int d^{4} \times \omega(x)\left\{d_{\alpha} \frac{\partial \mathscr{L}}{\partial \varphi_{\alpha}} \varphi_{\alpha}+d_{\alpha} \frac{\partial \mathcal{L}}{\partial \varphi_{\alpha, \lambda}} \varphi_{\alpha, \lambda}-d_{\alpha} \partial^{\lambda}\left(\frac{\partial \mathcal{L}}{\partial \varphi_{\alpha, \lambda}} \varphi_{\alpha}\right)\right\}
$$

The Lagrangian is a homogeneous expression of degree four except terms which break the dilatation invariance. Assuming that the only dimensional coupling constants are the masses, we can write

$$
\left(d_{\alpha}+i\right) \frac{\partial \mathcal{L}}{\partial \varphi_{\alpha, \lambda}} \varphi_{\alpha, \lambda}+d_{\alpha} \frac{\partial \mathcal{L}}{\partial \varphi_{\alpha}} \varphi_{\alpha}=4 \mathcal{L}-m_{\alpha} \frac{\partial \mathcal{L}}{\partial m_{\alpha}}
$$

where $m_{\alpha}$ is the mass of $\varphi_{\alpha}$. Substituting (2.25) into (2.24); we have

$$
\delta 1=-\int d^{4} x w(x)\left\{\vec{\theta}_{\mu}^{\mu}+\partial^{\lambda} v_{\lambda}+m_{\alpha} \frac{\partial \mathscr{L}}{\partial m_{\alpha}}\right\}
$$

where $\bar{\theta}_{\mu}^{\mu}$ is the trace of $\bar{\theta}_{\mu \nu}$ and 


$$
v_{\lambda}=d_{\alpha} \frac{\partial \mathcal{L}}{\partial \varphi_{\alpha,} \lambda} \varphi_{\alpha}-\frac{\partial \mathcal{L}}{\partial \varphi_{\alpha, \mu}}\left(\Sigma_{\mu \lambda}\right)_{\alpha \beta} \varphi_{\beta}
$$

Notice that $\vec{\theta}_{\mu}^{\mu}=\theta_{\mu}^{B \mu}$ from Eq. (2.17). $v_{\lambda}$ is called a field virial, ${ }^{14}$ and

$$
v_{\lambda}= \begin{cases}0 & \text { for fields of spin } \frac{i}{2} \text { and } 1 \\ \partial_{\lambda} \varphi \cdot \varphi & \text { for field of } \operatorname{spin} 0 .\end{cases}
$$

for Lagrangians without derivative couplings in a renormalizable theory. We shall restrict ourselves to the case $(2.28)$. We require that the additional term in Eq. (2.20), satisfies

$$
x_{\mu}^{\mu}=\partial^{\mu} v_{\mu}
$$

in order to obtain a proper trace relation for the improved energymomentum tensor, ie

$$
\theta_{\mu}^{\mu}+m_{\alpha} \frac{\partial \mathfrak{\xi}}{\partial m_{\alpha}}=0
$$

Equations (2.29) and (2.21) determine $x_{\mu \nu}$ uniquely

$$
x_{\mu \nu}= \begin{cases}0 & \text { for fields of spin } \frac{1}{2} \text { and } 1 \\ -\frac{1}{6}\left(\partial_{\mu} \partial_{\nu}-g_{\mu \nu} \partial^{\lambda} \partial_{\lambda}\right) \varphi^{2} \text { for field of spin } 0 .\end{cases}
$$

which satisfies the constraints listed in Eq. (2.21). Thus finally, we obtain 
$\theta_{\mu \nu}=\bar{\theta}_{\mu \nu}-\frac{c}{6}\left(\partial_{\mu} \partial_{\nu}-g_{\mu \nu} \partial^{\lambda} \partial_{\lambda}\right) \varphi^{2}$

where $c=1$ for field of spin 0 and $c=0$ for. fields of $\operatorname{spin} \frac{1}{2}$ and 1 . $\theta_{\mu \nu}$ is the improved energy-momentum tensor. 9

\section{F. Internal Symmetry Transformation}

The variations of fields defined in Eqs. $(2.2-2.4)$ can be applied to the cases of internal symmetry transformations. They are a generalization of the transformation proposed by Gell-Mann and Lévy, ${ }^{10}$ which corresponds to $G_{\alpha}^{\lambda[\mu]}=0$ in Eq. (2.3). In more general cases, however, gauge transformations contain the $G_{\alpha}^{\lambda[\mu]}$ term. (See, for example, the discussion of Gasiorowicz and Geffen ${ }^{15}$ on the Gauge transformation of chiral Lagrangians containing vector and axial-vector fields.) in the present paper, we will restrict ourselves to the case of $G_{\alpha}^{\lambda[\mu]}=0$ and will discuss it in Sec. 111 .

III. FUNCTIONAL INTEGRATION FORMULATION OF QUANTUM FIELD-THEORY

In this section, we review briefly the functional integration formulation of quantum field-theory. For definiteness, let us take an SU(3) symmetric model containing a triplet quark field $\psi(x)$ of mass $m$ interacting with a singlet vector gluon field $B_{\mu}(x)$ of mass $M$, for which we have a Lagrangian

$$
\begin{aligned}
\mathcal{L} & =\frac{1}{2} \bar{\psi}(x)\left(i \gamma^{\mu} \partial_{\mu}-m\right) \psi(x)+\frac{1}{2}\left(-i \partial_{\mu} \bar{\psi}(x) \gamma^{\mu}-m \bar{\psi}(x)\right) \psi(x) \\
& -\frac{1}{4} B_{\mu \nu}(x) B^{\mu \nu}(x)+\frac{M^{2}}{2} B_{\mu}(x) B^{\mu}(x) \\
& -g \bar{\psi}(x) \gamma^{\mu} \psi(x) B_{\mu}(x)
\end{aligned}
$$


with

$$
B_{\mu \nu}(x) \equiv \partial_{\mu} B_{\nu}(x)-\partial_{\nu \mu} B_{\mu}(x)
$$

The derivation discussed in Eqs. $(2.15-2.32)$ gives (note that $\theta_{\mu \nu}=\bar{\theta}_{\mu \nu}$ in the present case)

$$
\begin{aligned}
\theta_{\mu \nu}(x) & =\frac{1}{4}\left[\bar{\psi}(x) \gamma_{\mu} \partial_{\nu} \psi(x)-\partial_{\nu} \bar{\psi}(x) \gamma_{\mu} \psi(x)+\mu \leftrightarrow \psi\right] \\
& -B_{\mu \lambda}(x) B_{\nu}^{\lambda}(x)-\frac{1}{2}\left(\partial^{\lambda} B_{\lambda \mu}(x) B_{\nu}(x)+\mu \leftrightarrow \nu\right)-g_{\mu \nu} \mathscr{L}
\end{aligned}
$$

and by making use of equations of motion, we can prove the trace relation

$$
\theta_{\mu}^{\mu}(x)=\theta(x) \equiv m \bar{\psi}(x) \psi(x)-M^{2} B_{\mu}(x) B^{\mu}(x)
$$

which is just (2.30). For later use we list the su(3) currents, vector, axial-vector, scalar, and pseudoscalar, in this model

$$
\begin{aligned}
& V_{\mu}^{a}(x)=\bar{\psi}(x) \gamma_{\mu} \frac{\lambda_{a}}{2} \psi(x) \\
& A_{\mu}^{a}(x)=\bar{\psi}(x) \gamma_{\mu} \gamma_{5} \frac{\lambda_{a}}{2} \psi(x) \\
& S^{a}(x)=\bar{\psi}(x) \frac{\lambda_{a}}{2} \psi(x) \\
& P^{a}(x)=\bar{\psi}(x) i \gamma_{5} \frac{\lambda_{a}}{2} \psi(x)
\end{aligned}
$$

where $\lambda_{a}, a=0,1,2 \cdot \cdots 8$, are the Gell-Mann matrices.

In the functional integration formulation, ${ }^{4}$ the vacuum expectation value of a time-ordered product of $n$ functions of fields, $Q_{1}\left(x_{1}\right)$, . . $Q_{n}\left(x_{n}\right)$ is given by the following functional integral 


$$
\begin{aligned}
& \left\langle T^{*}\left(Q_{1}\left(x_{1}\right) \cdot \cdot Q_{n}\left(x_{n}\right)\right)\right\rangle_{0} \\
& \quad \equiv N^{-1} \int d \& D Q D B_{\mu} Q_{1}\left(x_{1}\right) \cdot \cdots Q_{n}\left(x_{n}\right) e^{i \prime},
\end{aligned}
$$

where' 1 is the action integral defined in Eq. (2.1) with $\mathcal{L}_{\text {given by }}$ Eq. (3.1). N is defined by

$$
N \equiv \int d \psi d Q \bar{\psi} d B_{\mu} e^{i I}
$$

and gives the vacuum amplitude. In Eq. (3.5), $Q_{i}(x)$ on the left-hand side represents a function of the fields as Heisenberg operators, while on the right-hand side it represents the same function of the fields as integration variables. The $\mathrm{T}^{*}$ symbol has dual meanings. First, it means the $T^{*}$-product of Koba and Nishijima., 16 in which derivatives of. operators inside a T-product are defined in terms of the dipole formula, i.e.

$$
\left\langle T^{*}\left(\partial_{\mu} \psi(x) \partial_{\nu} \psi(y) \cdot \cdot \cdot\right)\right\rangle_{0} \equiv \frac{\partial}{\partial x^{\mu}} \frac{\partial}{\partial y^{\nu}}\langle T(\psi(x) \psi(y) \cdot \cdot \cdot)\rangle_{0}
$$

This follows directly from the definition of the n-pf in terms of the functional integration (3.5). The symbol $\mathrm{T}^{*}$ stands also for covariantized T-product as in the case of the vector meson propagator. Notice that any S-matrix element can be expressed in terms of a functional integral of the form (3.5), if we take $Q_{i}(x)$ 's as asymptotic, in- or outfields.

In practical calculations, (3.5) can be evaluated by means of a generating functional defined as 


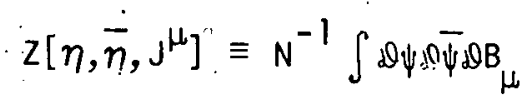

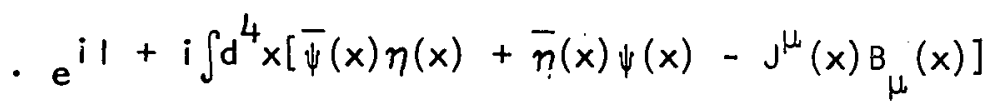

where $\bar{\eta}(\mathrm{x}), \eta \underline{(\mathrm{x})}$ and $\mathrm{J}^{\mu}(\mathrm{x})$ are external sources for the spinor and vector meson fields; and $\bar{n}(x), \eta(x), \psi(x)$ and $\bar{\psi}(x)$ anticommute with each other: Then

$$
\begin{aligned}
& \left\langle T^{*}\left(\psi(x) \bar{\psi}(y) B_{\mu}(z) \cdot \cdot \cdot\right)\right\rangle_{0} \\
& \quad=\left.\left(\frac{1}{i}\right)^{3} \frac{\partial}{\partial \bar{\eta}(x)} \frac{\partial}{\partial \eta(y)} \frac{\partial}{\partial J^{\mu}(z)} \cdot \cdots z\left[\eta, \bar{\eta}, J^{\mu}\right]\right|_{\eta=\bar{\eta}=J^{\mu}=0 .}
\end{aligned}
$$

Explicit forms of the generating functional will be derived in Appendix A. Let. us derive an axial-vector $W-T$ identity making use of the formulation of the functional integration. This serves as an illustration of the procedure which will be used over and over again in Sec. IV. We consider the transformations of fields

$$
\begin{aligned}
& \psi(x) \rightarrow\left(1+i \omega(x) \gamma_{5} \frac{\lambda_{a}}{2}\right) \psi(x) \\
& \bar{\psi}(x) \rightarrow \bar{\psi}(x)\left(1+i \omega(x) \gamma_{5} \frac{\lambda_{a}}{2}\right) \\
& B_{\mu}(x) \rightarrow B_{\mu}(x) .
\end{aligned}
$$

It is easy to show that under the change of variables (3.10),

$$
1 \rightarrow 1+\int d^{4} x \omega(x)\left[\partial^{\mu} A_{\mu}^{a}(x)-2 m p^{a}(x)\right]+0\left(\omega^{2}(x)\right) .
$$


For definiteness, let us derive the axial-vector $W-T$ identity for the AVV vertex. Consider

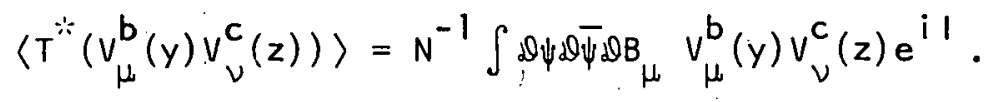

Changing the integration variables in Eq. (3.12) according to (3.10), the value of (3.12) is not changed as long as the integral exists, and we obtain

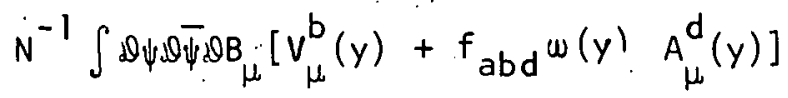

$$
\begin{aligned}
& {\left[V_{\nu}^{c}(z)+f_{\operatorname{acd}} w(z) A_{\nu}^{d}(z)\right] e^{i l+i \int d^{4} x w(x) \cdot\left[\partial^{\lambda} A_{\lambda}^{a}(x)-2 m p^{a}(x)\right]}} \\
& +0\left(\omega^{2}(x)\right)=\text { independent of } \omega(x),
\end{aligned}
$$

where the Jacobian of the transformation (3.10) is unity (see Appendix C). Differentiating (3.13) with respect to $\omega(z)$ and setting $\omega(z)$ to zero," we obtain

$$
\begin{aligned}
0= & N^{-1} \int D \psi \Delta Q \bar{\psi} D B_{\mu}\left\{f_{a b d} A_{\mu}^{d}(y) v_{\nu}^{c}(z) \delta^{4}(y-x)+f_{a c d} v_{\mu}^{b}(y) A_{\nu}^{d}(z) \delta(z-x)\right. \\
& \left.+i v_{\mu}^{b}(y) v_{\nu}^{c}(z)\left[\partial \lambda_{\lambda}^{a}(x)-2 m P^{a}(x)\right]\right\} e^{i l} .
\end{aligned}
$$

In the conventional notation this is

$$
\begin{aligned}
& \frac{\partial}{\partial x_{\lambda}}\left\langle T^{*}\left(A_{\lambda}^{a}(x) v_{\mu}^{b}(y) v_{\nu}^{c}(z)\right)\right\rangle_{0}=2 m\left\langleT ^ { * } \left( P^{a}(x) v_{\mu}^{b}(y) v_{\nu}^{c}(z)_{0}\right.\right. \\
& \quad+i f_{a b d}\left\langle T^{*}\left(A_{\mu}^{d}(y) v_{\nu}^{c}(z)\right)\right\rangle_{0} \delta^{4}(y-x)+i f_{a c d}\left\langle T^{*}\left(v_{\mu}^{b}(y) A_{\nu}^{d}(z)\right)\right\rangle_{0} \delta^{4}(z-x) .
\end{aligned}
$$

This is just the naive axial-vector $W-T$ identity for the AVV vertex. 
With Eqs. (3.9) and Eq. (A.16) or (A.24), a perturbation expansion can be obtained for an n-pf. The result is identical to that of the conventional approach. In the following we give a brief consideration of a regularization scheme to eliminate divergences of the theory. We shall follow the scheme of Pauli-Villars-Gupta. 17 In addition to the physical spinor fields $\psi$ and $\bar{\psi}$ and the vector fields $B_{\mu}$, we introduce one auxiliary vector field $B_{\mu}^{R}$ of mass $M_{R}$ and $n$ auxiliary fermion fields $\psi_{1}, \psi_{2}, \ldots, \ldots, \psi_{n}$ of mass $m_{1}, m_{2}, \ldots ., m_{n}$ respectively. We denote the physical spinor field and its mass by $\psi_{0}$ and $m_{0}$. In the end, $M_{R}$ and $m_{l}, l=1,2, \ldots . n$, go to infinity. . The Lagrangian is

$$
\begin{aligned}
\mathcal{L}(x) & =\frac{1}{2} \sum_{l=0}^{n}\left\{\bar{\psi}_{l}(x)\left(i \vec{z}-m_{l}\right) \psi_{l}(x)+\bar{\psi}_{l}(x)(-i \bar{z}-m) \psi_{l}(x)\right\} \\
& -\frac{1}{4} B_{\mu \nu}(x) B^{\mu \nu}(x)+\frac{1}{2} M^{2} B_{\mu}(x) B^{\mu}(x)-\frac{1}{4} B_{\mu \nu}^{R}(x) B^{R}(x)^{\mu \nu} \\
& +\frac{1}{2} M_{R}^{2} B_{\mu}^{R}(x) B^{R \mu}(x)-g \sum_{l=0}^{n} \bar{\psi}_{l}(x) \gamma^{\mu} \psi_{l}(x)\left(B_{\mu}(x)+B_{\mu}^{R}(x)\right) \\
& +\Sigma_{\alpha} j_{\alpha}(x) \Gamma^{\alpha}(x)
\end{aligned}
$$

where $B_{\mu \nu}^{R}(x)$ is defined similar to $B_{\mu \nu}(x)$ (see Eq. (3.1)).

$\mathrm{j}_{\alpha}(\mathrm{x})$, with $\alpha$ representing the internal symmetry and Lorentz tensor indices, represent Su(3) currents, energy-momentum tensor, and its trace, their explicit expressions are given previously in this section. Now, they contain also the regulator field, for example, the vector current, etc, are

$$
j_{\alpha}(x)=\sum_{l=0}^{n} \bar{\psi}_{l}(x) \gamma_{M} \frac{\lambda_{a}}{2} \psi_{l}(x)
$$

etc. $\Gamma^{\alpha}(x)$ are external currents having the same tensorial properties as 
those of $j \alpha(x)$. Unlike the conventional approach in which signs of the terms involving a certain regulator fields are opposite to that of the physical fields, their signs in Eq. (3.1b) are the same. This allows us to introduce a small negative imaginary part to all masses involved in order to obtain the Feynman Propagators and maintain the convergence of the functional integral. The regularization can be achieved by requiring a certain regulator fields $\psi_{j}$ to be commuting fields rather than anti-commuting fields. This corresponds to requiring those fields to satisfy Bose statistics in the quantized field version. Following, Lee, 17 we assign signature factors $c_{\ell}=+1(-1)$ to an anti-commuting (communting) fermion fields. $m_{l}, c_{l}$, and $n$ are chosen to satisfy

$$
\begin{aligned}
& \sum_{l=0}^{n} c_{l}{ }_{m_{l}^{\alpha}}^{\alpha}=0 \quad \alpha=0 ; 1,2 \\
& c_{0}=1
\end{aligned}
$$

but otherwise unspecified. We write the generating functional

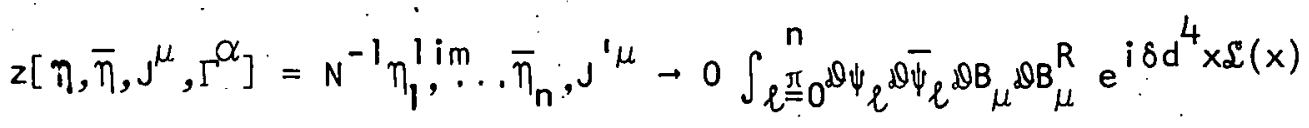

$$
\begin{aligned}
& \text { - e } \quad \sum_{\ell=0}^{n} d^{4} x\left[\bar{\eta}_{l}(x) \psi_{l}(x)+\bar{\psi}_{\ell}(x) \eta_{l}(x)\right] \\
& -i \int d^{4} x\left[J^{\mu}(x) B_{\mu}(x) J^{\prime} \mu(x) B_{\mu}^{R}(x)\right] \\
& e^{i \Sigma \int d^{4} x \Gamma^{\alpha}(x)} j_{\alpha}(x)
\end{aligned}
$$

where $\eta \equiv \eta_{0}$ and $\bar{\eta} \equiv \bar{\eta}_{0} ; \eta_{\ell}$ and $\bar{\eta}_{\ell}$ are taken to be anti-commuting (commuting), if the signature factor of the corresponding field is $c_{l}=+1 \quad\left(c_{\ell}=-1\right)$. 
Notice that the auxiliary vector meson field couples to the external source through an imaginary coupling relative to that of the vector meson field itself. This may look to destroy the very existence of the functional integration in (3.17), but it actually does not do so. For in order to obtain Feynmann rules, we have to assign small negative imaginary parts to all masses in the Lagrangian (see Appendix A), which in turn gives a negative real part $\varepsilon \int\left(B_{\mu} B^{\mu}+B_{\mu}{ }^{R} B^{R} y\right) d x$ in the exponents $i \int \mathcal{L}_{j}(x) d^{4} x$, providing a convergent factor to the integrand of the functional integration. Since this term is quadratic in $B_{\mu}^{R}$, it dominates over the linear coupling term $\int_{\mu} B^{\mu}$. [The above argument shows that $B_{0}$ must be treated as an imaginary integration variable.] In Appendix A, we shall derive an explicit form for the generating functional (3.18).

\section{WARD-TAKAHASHI IDENTITIES AND TRACE RELATIONS}

Derivation of $W-T$ identities and trace relations in the functional integration method is straight-forward once the transformation of fields described in Sec. II are used as change of integration variables and the Jacobians of the transformations are obtained. The simplicity of the present approach is due to the fact that the use of equations of motion and equal time commutation relations which are essential in the conventional method can be avoided. The essential steps for deriving W-T identities have aiready been illustrated in Sec. 111 and in Popov and Faddeev ${ }^{4}$ and Lee and Zinn-Justin. 5 


\section{A. Current W-T Identities}

By current $W-T$ identity, we mean those with respect to the currents e.g.

$$
\frac{\partial}{\partial x_{\mu}}\left\langle T^{*}\left(j_{\mu}^{a}(x) \theta_{\lambda p}(z) j_{\nu_{1}}^{b}\left(y_{1}\right) \cdots \cdot j_{\nu_{n}}^{b}\left(y_{n}\right)\right)\right\rangle
$$

where $j_{\nu_{i}}^{b}{ }_{i}\left(x_{i}\right), i=1,2, \cdots n$, are vector, axial-vector, scalar, or pseudoscalar currents; $b_{i}$ are SU(3) indices; $\nu_{i}$ represents the Lorentz indices in the case of vector and axial-vector currents. The forms of the currents are given in Eqs. $(3.4 a-d)$. Our starting point is the $n+1-p f$ defined by

$$
\begin{aligned}
& \left\langle T^{* *}\left(\theta_{\lambda p}(z) \cdot j_{\nu_{1}}^{b}\left(y_{1}\right) \cdot \cdots \cdot j_{\nu_{n}}^{b}\left(y_{n}\right)\right)\right\rangle \\
& \equiv N^{-1} \int d \psi \Delta \theta \psi \bar{\psi}_{\mu} B_{\mu}\left[\theta_{\lambda_{0}}(z) j_{\nu_{1}}^{b}\left(y_{1}\right) \cdots j_{\nu_{n}}^{b}\left(y_{n}\right)\right] e^{i l} .
\end{aligned}
$$

\section{Axial-Vector $W-T$ Identities. Here}

$$
j_{\mu}^{a}(x)=A_{\mu}^{a}(x)
$$

We consider the change of variables (3.10) in (4.2)

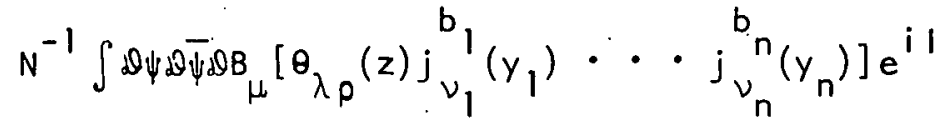

$$
\begin{aligned}
& =N^{-1} \int d 0 \psi D \bar{\psi} \overline{D B}_{\mu}\left[\left(\theta_{\lambda \rho}(z)+\delta \theta_{\lambda \rho}(z)\right)\left(j_{\nu_{1}}^{b}\left(y_{1}\right)+\delta j_{\nu_{1}}^{b}\left(y_{1}\right)\right) \cdot \cdots\right. \\
& \left.\left(j_{\dot{\nu}_{n}}^{b}\left(y_{n}\right)+\delta j_{\nu_{n}}^{b}\left(y_{n}\right)\right) e^{i l+i \delta l}\right],
\end{aligned}
$$

where the Jacobian of the transformation is unity and to the first order in $\omega(x)$, the variations $\delta 1$, etc. are 


$$
\begin{aligned}
& \dot{\delta 1}=\int d^{4} x \omega(x)\left[\partial^{\mu} A_{\mu}^{a}(x)-2 m P^{a}(x)\right] \\
& \delta A_{\nu}^{b}(x)=w(x) f_{a b c} v_{\nu}^{c}(x) \\
& \delta V_{\nu}^{b}(x)=w(x) f_{a b c} \cdot A_{\nu}^{c}(x) \\
& \delta S^{b}(x)=w(x) d_{a b c} P^{c}(x) \\
& \delta P^{b}(x)=-\omega(x) d_{a b c} S^{c}(x) \\
& \delta \theta_{\mu \nu}(x)=g_{\mu \nu}\left[\partial \lambda_{\omega}(x) A_{\lambda}^{a}(x)+2 m \omega(x) P^{a}(x)\right] \\
& -\frac{1}{\partial}\left[\partial_{\mu} \omega(x) A_{\nu}^{a}(x)+\partial_{\nu} \omega(x) A_{\mu}^{a}(x)\right]
\end{aligned}
$$

Since the left-hand side of Eq. (4.3) is independent of $\omega(x)$, we obtain after differentiating it with respect to $\omega(x)$ and setting $\omega(x)=0$

$$
\begin{aligned}
& 0=N^{-1} \int Q \psi d Q \psi \mathcal{H} B B_{\mu}\left\{\left[\left(-\frac{1}{2}\left(A_{\lambda}^{a}(z) \partial_{\rho} \delta(z-x)+A_{\rho}^{a}(z) \partial_{\lambda} \delta(z-x)\right)\right.\right.\right. \\
& \left.+g_{\lambda \rho}\left(A_{\mu}^{a}(z) \partial^{\mu} \delta(z-x)+2 m P^{a}(z) \delta(z-x)\right) j_{\nu_{1}}^{b}\left(y_{1}\right) \cdots \cdot j_{\nu_{n}}^{b}\left(y_{n}\right)\right]
\end{aligned}
$$

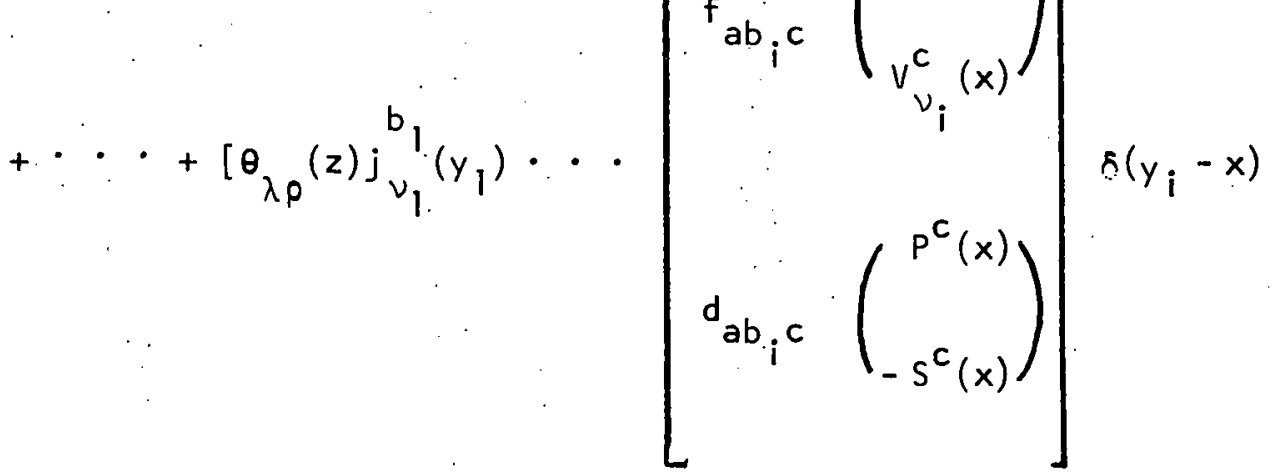

$$
\begin{aligned}
& \left.\cdots j_{\nu_{n}}^{b}\left(y_{n}\right)\right]+\cdots \cdot \\
& \left.+i\left[\partial^{\mu} A u^{a}(x)-2 m P^{a}(x)\right] j_{\nu_{1}}^{b_{1}}\left(y_{1}\right) \cdot . \cdot j_{\nu_{n}}^{b_{n}}\left(y_{n}\right)\right\} e^{i I}
\end{aligned}
$$


The four cases in the second term correspond to $j_{\nu_{i}}^{b}$ being a vector; an axial-vector, a scalar, or a pseudoscalar current. Eq. (4.5) leads to the $W-T$ identity

$$
\begin{aligned}
& -i \frac{\partial}{\partial x_{\mu}}\left\langle T^{*}\left(A_{\mu}^{a}(x) \theta_{\lambda \rho}(z) j_{\nu_{1}}^{b}\left(y_{1}\right) \cdot j_{\nu_{n}}^{b}\left(y_{n}\right)\right)\right\rangle_{0} \\
& =-2 m i\left\langle T^{*}\left(P^{a}(x) \theta_{\lambda \rho}(z) j_{\nu_{1}}^{b}\left(y_{1}\right) \cdot j_{\nu_{n}}^{b}\left(y_{n}\right)\right)\right\rangle_{0} \\
& -\frac{1}{2}\left(\delta_{\rho}^{\top} \delta_{\lambda}^{\sigma}+\delta_{\rho}^{\sigma} \delta_{\lambda}^{\tau}-{ }^{2 g}{ }_{\lambda \rho} g^{\tau \sigma}\right) \frac{\partial}{\partial z} \delta(z-x) \cdot\left\langle T^{*}\left(A_{\sigma}^{a}(z) j_{\nu_{1}}^{b}\left(y_{1}\right) \cdot \cdot \cdot j_{\nu_{n}}^{n_{n}}\left(y_{n}\right)\right)\right\rangle_{o} \\
& +2 m \delta(z-x) g_{\lambda \rho}\left\langle T^{*}\left(p^{a}(z) j_{\nu_{1}}^{b}\left(y_{1}\right) \cdots \cdot j_{\nu_{n}}^{b}\left(y_{n}\right)\right)\right\rangle_{0}+\cdots \cdot \\
& +\delta\left(y_{i}-x\right)\left\langleT ^ { * } \left(\theta_{\lambda \rho}(z) j_{\nu_{1}}^{b}{ }^{1}\left(y_{1}\right) \ldots .\right.\right. \\
& d_{a b_{i} c} \quad\left(\begin{array}{c}
p^{c}(x) \\
s^{c}(x)
\end{array}\right) \\
& \left.\left.\cdot \cdot j_{\nu_{n}}^{b}\left(y_{n}\right)\right)\right\rangle_{0} \\
& +\cdot \cdot \cdot
\end{aligned}
$$

When calculated according to Eq. (3.9) in a perturbation expansion, Eq. (4.6) contains totally connected parts as. well disconnected ones involving products of two or more totally connected m-point function, $m \leq n$. Therefore, Eq. (4.6) contains the $W-T$ identities of the totally 
connected $(n+2)$-point function, $(n+1)$-point function, etc. This decomposition occurs in the conventional approach as a result of the Wick theorem. It is easy to convince oneself that the W-T identities of these connected $(n+2)-p f,(n+1)-p f$, etc. are satisfied individually. Therefore, we consider the W-T identity of an $n-p f$ to contain only totally connected parts for all the terms involved.

Vector $W-T$ Identities. The transformations for vector $W-T$ identities are

$$
\begin{aligned}
& \psi(x) \rightarrow\left(1+i \omega(x) \frac{\lambda_{a}}{2}\right) \psi(x) \\
& \bar{\psi}(x) \rightarrow \bar{\psi}(x)\left(1-i \omega(x) \frac{\lambda_{a}}{2}\right) \\
& B_{\mu}(x) \rightarrow B_{\mu}(x)
\end{aligned}
$$

which lead to the following variations to first order in $\omega(x)$.

$$
\begin{aligned}
\delta I & =\int d^{4} x \omega(x) \partial^{\mu} v_{\mu}^{a}(x) \\
\delta j_{\nu}^{b}(x) & =\omega(x) f_{a b c} j_{\nu}^{c}(x) \\
\delta \theta & \\
{ }_{\lambda \rho}(x) & =-\frac{1}{2}\left[\partial_{\rho}^{\omega}(x) v_{\lambda}^{a}(x)+\partial_{\lambda} \omega(x) v_{\rho}^{a}(x)\right]+g_{\lambda \rho} \partial^{\mu} \omega(x) v_{\mu}^{a}(x)
\end{aligned}
$$

where $j_{v}^{b}(x)$ is any of the four currents. Again the Jacobian of the transformations (4.7) is unity and we obtain the W-T identity 


$$
\begin{aligned}
& -i \frac{\partial}{\partial x_{\mu}}\left\langle T^{* *}\left(v_{\mu}^{a}(x) \theta_{\lambda \rho}(z) j_{\nu_{1}}^{b}\left(y_{1}\right) \cdot j_{\nu_{n}}^{b}\left(y_{n}\right)\right)\right\rangle_{0} \\
& =-\frac{1}{2}\left(\delta_{\lambda}^{\sigma} \delta_{\rho}^{\tau}+\delta_{\rho}^{\sigma} \delta_{\lambda}^{\tau}-2 g_{\lambda \rho} g^{\sigma \tau}\right) \frac{\partial}{\partial z^{\sigma}} \delta(z-x) \cdot\left\langle T^{*}\left(v_{T}^{a}(x) j_{\nu_{1}}^{b}\left(y_{1}\right) \cdot \cdot \cdot j_{\nu_{1}}^{n_{n}}\left(y_{n}\right)\right)\right\rangle_{o} \\
& +\cdots+f_{a b} c^{c}\left\langle T^{*}\left(\theta_{\lambda \rho}(z) j_{\nu_{1}}^{b}\left(y_{1}\right) \cdots \cdot j_{\nu_{i}}^{c}\left(y_{i}\right) \cdots \cdot j_{\nu_{n}}^{b_{n}}\left(y_{n}\right)\right)\right\rangle_{0} \delta\left(y_{i}-x\right) \\
& +\cdot \cdot
\end{aligned}
$$

\section{B. Energy-Momentum Tensor $W-T$ Identity}

We consider

$$
\frac{\partial}{\partial z \lambda}\left\langle T^{*}\left(\theta_{\lambda \rho}(z) j_{\nu_{1}}^{b}\left(y_{1}\right) \cdot: \cdot j_{\nu_{n}}^{b}\left(y_{n}\right)\right)\right\rangle_{0^{*}}
$$

The transformation of the field variables are, according to (2.18)

$$
\begin{aligned}
& \psi(x) \rightarrow\left(1+\omega^{\lambda}(x) \partial_{\lambda}+\frac{1}{8} \partial \lambda_{\omega}^{\rho}(x)\left[\gamma_{\lambda}, \gamma_{\rho}\right]\right) \psi(x) \\
& \bar{\psi}(x) \rightarrow \bar{\psi}(x)\left(1+\overleftarrow{\partial}_{\lambda} \omega^{\dot{\lambda}}(x)-\frac{1}{8} \partial^{\lambda}{ }_{\omega}^{\partial}(x)\left[\gamma_{\lambda}, \gamma_{\rho}\right]\right) \\
& B_{\mu}(x) \rightarrow\left(g_{\mu \nu}+g_{\mu \nu} \omega^{\lambda}(x) \partial_{\lambda}+\frac{1}{2} \partial^{\lambda}{ }_{\omega} \rho\left[g_{\lambda \mu} g_{\rho \nu}-g_{\lambda \nu} g_{\rho \mu}\right]\right) B^{\nu}(x)
\end{aligned}
$$

for which the Jacobian is unity (see Appendix C). Transformations (4.11) generate the following variations.

$$
\delta j_{\mu}^{a}(x)=\omega^{\lambda}(x) \partial_{\lambda} j_{\mu}^{a}(x)+\frac{1}{8} \partial^{\lambda} \omega^{\rho}(x)\left(\bar{\Sigma}_{\lambda \rho}\right)_{\mu \nu \nu} j^{a}(x)^{\nu}
$$

for the four types of currents; where 


$$
\left(\bar{\Sigma}_{\lambda \rho \mu \nu}\right)= \begin{cases}g_{\lambda \mu} g_{\rho \nu}-g_{\lambda \nu} g_{\rho \mu} \text { for vector and axial-vector currents } \\ 0 \quad \text { for scalar and pseudoscalar currents. }\end{cases}
$$

Making variable change (4.11) in the following $n-p f$

$$
N^{-1} \int \Delta \psi \Delta \psi \Delta B B_{\mu}\left[j_{\nu_{1}}^{b}\left(y_{1}\right) \cdot \cdots \cdot j_{\nu_{n}}^{b}\left(y_{n}\right)\right] e^{i l}
$$

we obtain, by the same procedure described in deriving the current W-T identity,

$$
\begin{aligned}
& i \frac{\partial}{\partial x}\left\langle T^{*}\left(\theta_{\lambda \rho}(x) j_{\nu_{1}}^{b}\left(y_{1}\right) \cdot \cdot \cdot j_{\nu_{n}}^{b}\left(y_{n}\right)\right)\right\rangle_{0} \\
& =\cdot \cdot+\left[\delta_{\nu_{i}}^{\top} \delta\left(x-y_{i}\right) \frac{\partial}{\partial y_{i} \rho}+\frac{1}{z}\left(\bar{\Sigma}_{\lambda \rho}\right)^{\rho} \nu_{i}{ }^{\top} i\left(\frac{\partial}{\partial y_{i \lambda}} \delta\left(y_{i}-x\right)\right)\right] . \\
& \left\langle T^{*}\left(j_{\nu_{1}}^{b}{ }^{b}\left(y_{1}\right) \cdot \cdot j_{T_{i}}^{b}{ }_{i}\left(y_{i}\right) \cdot j_{\nu_{n}}^{b}\left(y_{n}\right)\right)\right\rangle_{0}+\cdots \cdot \cdot
\end{aligned}
$$

C. Trace Identity

We apply to the n-pf, Eq. (4.13), the transformation (2.22), i.e. 


$$
\begin{aligned}
& \psi(x) \rightarrow(1+3 / 2 \omega(x)) \psi(x) \\
& \bar{\psi}(x) \rightarrow(1+3 / 2 \omega(x)) \bar{\psi}(x) \\
& B_{\mu}(x) \rightarrow(1+\omega(x)) B_{\mu}(x)
\end{aligned}
$$

which induce the variations

$$
\delta j_{\mu}^{a}(x)=3 j_{\mu}^{a}(x)
$$

for the four types of currents; and

$$
\delta 1=-\int d^{4} x\left[\theta_{\mu}^{\mu}(x)-\theta(x)\right] \omega(x)
$$

where $\theta_{\mu}^{\mu}(x)$ and $\theta(x)$ are given by Eqs. (3.2) and (3.3) respectively. Let us denote the Jacobian of the transformations $(4.15)$ by $J(\omega)$

$$
J(w) \equiv J_{\psi}(w) J_{\psi}(\omega) j_{B}(w)
$$

where $j_{\psi}(x)$, etc. are the Jacobians of the transformations for $\psi$ etc. The n-pf (4.14) becomes

$$
\begin{aligned}
& J(\omega) N^{-1} \int d Q \psi \partial \nabla \alpha B_{\mu}\left[\left(j_{\nu_{1}}^{b}\left(y_{1}\right)+\delta j_{\nu_{1}}^{b}\left(y_{1}\right)\right) \cdot \cdots\left(j_{\nu_{n}}^{b}\left(y_{n}\right)+\delta j_{\nu_{n}^{n}}^{b}\left(y_{n}\right)\right)\right] . \\
& \text { - } e^{i 1+i \delta 1} \text {. }
\end{aligned}
$$

Differentiating it with respect to $\omega(x)$ and setting $\omega(x)=0$, we obtain 


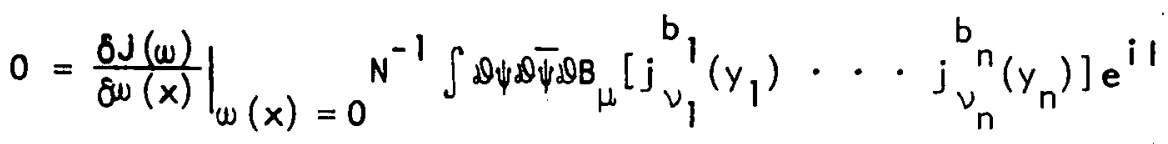

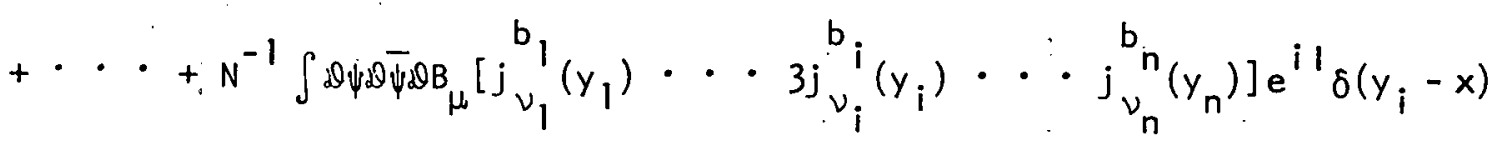

$$
\begin{aligned}
& +\cdot \cdot \\
& -i \int d \psi d \psi d B B_{\mu}\left[j_{\nu_{1}}^{b}\left(y_{1}\right) \cdot \cdot j_{\nu_{n}}^{b_{n}}\left(y_{n}\right)\left(\theta_{\mu}^{\mu}(x)-\theta(x)\right)\right] e^{i l} .
\end{aligned}
$$

In the above expression, we have used $J(0)=1$. Take first $n=0$, we obtain

$$
\frac{\delta J(\omega)}{\delta \omega(x)}-i\left\langle T^{*}\left(\theta_{\mu}^{\mu}(x)-\theta(x)\right)\right\rangle_{0}=0
$$

Notice that the second term does not vanish in spite of the trace relation (3.3). This is because that $\theta_{\mu}^{\mu}(x)-\theta(x)$, bilinear in fields, is defined by the functional integration as their $\mathrm{T}^{*}$-products which in turn, dictates that derivatives be outside the T-product. Thus the equations of motion do not apply directly on fields inside the T-product and hence Eq. (3.3) can not be used. In Appendix B, we shall prove (4.19) by an explicit evaluation. For $n$ greater than zero; we separate successively disconnected parts with the aid of (4.19), we obtain finally the trace identity

$$
\begin{aligned}
g^{\lambda \rho}\left\langle T^{*}\left(\theta_{\lambda \rho}(x) j_{\nu_{1}}^{b}\left(y_{1}\right) \cdots \cdot j_{\nu_{n}}^{b}\left(y_{n}\right)\right)\right\rangle_{0} \\
=\left\langle T^{*}\left(\theta(x) j_{\nu_{1}}^{b}\left(y_{1}\right) \cdots \cdot j_{\nu_{n}}^{b}\left(y_{n}\right)\right)\right\rangle_{0} \\
\quad-3 i\left[\delta^{4}\left(y_{1}-x\right)+\cdots \cdot \delta^{4}\left(y_{n}-x\right)\right]\left\langle T^{*}\left(j_{\nu_{1}}^{b}\left(y_{1}\right) \cdot \cdots j_{\nu_{n}}^{b}\left(y_{n}\right)\right)\right\rangle_{o}
\end{aligned}
$$

Ward-Takahashi identities with respect to currents and involving the trace of the energy-momentum tensor can be obtained easily. We shall not list them here. 
Although all the relations derived so far in this section are in terms of the vacuum expectation values, they are valid between any physical states. This is because that any number of the $j_{\nu}^{b}(x)^{\text {'s }}$ can be substituted by asympototic fields at $\left|x_{0}\right| \rightarrow \infty$; thus the vacuum expectation values are converted into matrix elements between physical states. Detailed argument of this type will be given in Sec. $V$.

A few examples of the $W-T$ identities and trace identities which have been derived from quite different methods in the 1 iterature ${ }^{8}$ are listed below. Let

$$
J_{\mu}(x)=v_{\mu}^{3}(x)+\frac{1}{\sqrt{3}} v_{\mu}^{8}(x)
$$

be the electromagnetic current. From (4.9); (4.14), and (4.20) we obtain respectively

$$
\begin{aligned}
& i \frac{\partial}{\partial y}\left\{T_{\mu}^{*}\left(\theta_{\lambda \rho}(x) J_{\mu}(y)\right\}\right. \\
& =\frac{1}{2} J^{\sigma}(x)\left[g_{\lambda \sigma} \frac{\partial}{\partial x}+g_{\rho \sigma} \frac{\partial}{\partial x^{\lambda}}-2 g_{\lambda \rho} \frac{\partial}{\partial x^{\sigma}}\right] \delta(x-y) \\
& i \frac{\partial}{\partial x_{\lambda}}\left\{T^{*}\left(\theta_{\lambda \rho}(x) J_{\mu}(y)\right\}\right. \\
& =\frac{1}{2}\left\{2 g_{\mu \nu} \delta(y-x) \frac{\partial}{\partial y^{\rho}}+\left(g_{\lambda \mu} g_{\rho \nu}-g_{\lambda \nu} g{ }^{\rho}\right) \frac{\partial}{\partial y} \delta(y-x)\right\} J^{\nu}(y),
\end{aligned}
$$

and

$$
\begin{aligned}
& g^{\lambda \rho}\left\{T^{*}\left(\theta_{\lambda \rho}(x) J_{\mu}(y)\right)\right\} \\
& =T^{*}\left(\theta(x) J_{\mu}(y)\right)-3 i \delta(x-y) J_{\mu}(y) .
\end{aligned}
$$


!e shall also give the following two relations between the energymomentum tensor and the fermion fields

$$
\begin{gathered}
i \frac{\partial}{\partial x^{\lambda}}\left\{T^{*}\left(\theta_{\lambda \rho}(x) \psi_{\alpha}(y) \bar{\psi}_{\beta}(z)\right\}\right. \\
=\left[\delta(y-x) \frac{\partial}{\partial y^{\rho}}+\delta(z-x) \frac{\partial}{\partial z^{\rho}}\right]\left\{T^{*}\left(\psi_{\alpha}(y) \bar{\psi}_{\beta}(z)\right)\right\} \\
+\frac{1}{8}\left\{T^{*}\left(\psi_{\alpha^{\prime}}(y) \psi_{\beta^{\prime}}(z)\right)\right\}\left\{\left[\gamma_{\lambda}, \gamma_{\rho}\right]_{\alpha \alpha^{\prime}} \delta_{\beta \beta^{\prime}} \frac{\partial}{\partial y} \delta(y-x)\right. \\
\left.-\left[\gamma_{\lambda}, \gamma_{\rho}\right]_{\beta^{\prime} \beta} \delta_{\alpha \alpha^{\prime}} \frac{\partial}{\partial z} \delta(z-x)\right\}
\end{gathered}
$$

and

$$
\begin{aligned}
& \operatorname{ig}^{\lambda \rho}\left\{T^{*}\left(\theta_{\lambda \rho}(x) \psi(y) \bar{\psi}(z)\right)\right\} \\
& \quad=i T^{*}(\theta(x) \psi(y) \bar{\psi}(z))+\frac{3}{2}\left\{T^{*}(\psi(y) \bar{\psi}(z))\right\}\{\delta(y-x)+\delta(z-x)\} .
\end{aligned}
$$

Using the Feynman rules given in Appendix $C$, we have checked that, to the lowest (zeroth) order in the spinor-gluon coupling constant, all the W-T identities, $(4.6),(4.9),(4.14),(4.21),(4.23)$, and $(4.24)$, and the trace identities, (4.20) and (4.25), are satisfied naively. By "naively," we mean this, in evaluating the loop integrations we have freely shifted the origin of the integration variables, ignoring the surface terms due to the shift. We shall comment on this again in Sec. VI. 
V. DERIVATION OF CONSERVATION LAWS AND COMMUTATION RELATIONS

FROM W-T IDENTITIES

In the derivation of $W-T$ identities using conventional method, relevent conservation (or partial conservation) laws and equal time commutators, as operator relations, are needed. The present method does not resort to this intermediate knowledge, although we did use the same field transformations which give conservation laws from the action principle (see Sec. III). Two questions arise naturally in the construction of a field-theory soley using the functional integration method: (1) Do conservation laws follow from W-T identities? (2) Are equal-time commutators among currents and fields derivable from W-T identities? The second question is meaningful only when the equality is assumed between the n-point functional integral, in which fields are simply c-number integration variables, and the operator $n-p f$ as expressed in Eq. (3.5). In the following, we give a brief consideration to the above questions, for which we have positive answers.

To illustrate, let us deduce $\partial^{\mu} \theta_{\mu \nu}(x)=0$. Consider (4.24), we obtain for finite $x_{\mu}$,

$$
\begin{aligned}
& \lim _{y_{0} \rightarrow \infty} \frac{\partial}{\partial x_{\lambda}}\left\langle T^{*}\left(\theta_{\lambda \rho}(x) \psi(y) \bar{\psi}(z)\right)\right\rangle \\
& z_{0} \rightarrow-\infty \\
& =\lim _{y_{0} \rightarrow \infty}\left\langle T^{*}\left(\frac{\partial}{\partial x_{\lambda}} \theta_{\lambda \rho}(x) \psi(y) \bar{\psi}(z)\right)\right\rangle=0 . \\
& z_{0} \rightarrow-\infty
\end{aligned}
$$

The seagull terms which covariantize the T-product do not contribute in the above limit, since they contain at least one $\delta$-function involving the 
the arguments of two of the operators in the T-product. ${ }^{18}$. If there are more than three operators, the passing to the limit of infinite times can be carried out successively, so that none of the $\delta$-functions in the time. coordinates will survive. Eq. (5.1) is simply the matrix element of $\partial^{\lambda} \theta_{\lambda \rho}(x)$ between one fermion states. We can generalize Eq. (5.1) to more general cases and deduce the following relation

$$
\left\langle n \text {, out }\left|\partial^{\mu} \theta_{\mu \nu}(x)\right| n^{\prime}, \text { in }\right\rangle=0
$$

for arbitrary $\mid n$, out $\rangle$ and $\mid n^{\prime}$, in $\rangle$. Clearly (5.2) is equivalent to the usual statement of the conservation of the energy-momentum tensor as an operator equation.

The derivation of equal-time commutation relations is slightly more involved. Let us first consider a simple case, the equal-time commutator between the time component of a vector or axial-vector current and an arbitrary component of one of the four types of current. Let us take a current W-T identity similar to $(4.10)$ without the energy-momentum tensor

$$
-i \frac{\partial}{\partial x_{\mu}} T^{*}\left(v_{\mu}^{a}(x) j_{\nu}^{b}(y)\right)=f_{a b c} j_{\nu}^{c}(y) \delta^{4}(y-x),
$$

where $j_{\nu}^{b}(y)$ represents one of the four currents. This relation holds between any physical state as explained above. Further, the conservation of vector current $\partial^{\mu} v_{\mu}^{a}(x)=0$ follows by letting $y_{o} \rightarrow \infty$ and $x_{\mu}$ arbitrary but finite. We obtain immediately

$$
\delta\left(x_{0}-y_{0}\right)\left[v_{o}^{a}(x), j_{\nu}^{b}(y)\right]=i f_{a b c} j_{\nu}^{c}(y) \delta^{4}(y-x)+\text { Schwinger term. }
$$

The Schwinger term can not be determined in the present approach; it is intimately related to the seqgull term which covariantizes the T-product. 
We can also derive the canonical equal-time commutator of the fermion fields. 19 Let us consider

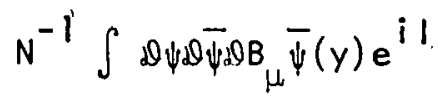

and make the field transformations which generate the equation of motion for $\psi(x)$

$$
\begin{aligned}
& \psi(x) \rightarrow \psi(x) \\
& \bar{\psi}(x) \rightarrow \bar{\psi}(x)+\bar{w}(x) \\
& B_{\mu}(x) \rightarrow B_{\mu} .
\end{aligned}
$$

Then, the variation of the action integral is

$$
\delta 1=\int d^{4} x \bar{\omega}(x)\left[i \gamma^{\mu} \partial_{\mu}-m-g \gamma^{\mu} B_{\mu}(x)\right] \psi(x)
$$

Substituting (5.5) and (5.6) into (5.4) and using the fact that the Jacobian for the transformation (5.5) is one, we obtain

$$
\left.-i\left\langle T^{*}\left(i \gamma^{\mu} \frac{\partial}{\partial x^{\mu}}-m-g \gamma^{\mu} B_{\mu}(x)\right) \psi(x) \bar{\psi}(y)\right)\right\rangle_{o}=\delta^{4}(y-x) .
$$

Taking $y_{0} \rightarrow \infty$ and $x_{\mu}$ finite, we obtain

$$
\left(i \gamma^{\mu} \partial_{\mu}-m-g \gamma^{\mu}{ }_{\mu}(x)\right) \psi(x)=0
$$

between the vacuum and one fermion state. Again one can obtain Eq. (5.8) between any physical states; it is, needless to say, the equation of motion of $\psi(x)$. Now, using Eq. (5.8) as an identity in Eq. (5.7), we get 


$$
\left\{y^{o} \psi(x), \bar{\psi}(y)\right\}+\delta\left(x_{0}-y_{0}\right)=\delta^{4}(x-y)
$$

which is the familiar canonical equal-time commutator. The equal-time commutator of the vector meson field can be derived in a similar way. Consider the variation of fields

$$
\begin{aligned}
& \psi(x) \rightarrow \psi(x), \\
& \bar{\psi}(x) \rightarrow \bar{\psi}(x) \\
& B_{\mu}(x) \rightarrow B_{\mu}(x)+\omega_{\mu}(x)
\end{aligned}
$$

which give rise to a variation of the action integral:

$$
\delta l=\int d^{4} x \omega^{\mu}(x)\left\{\left[g_{\mu \nu}\left(\partial^{\rho} \partial_{\rho}+\dot{M}^{2}\right)-\partial_{\mu} \partial_{\nu}\right] B^{\nu}(x)-g \bar{\psi}(x) \gamma_{\mu} \psi(x)\right\}
$$

Substituting $(5.10)$ and $(5.11)$ into the following integral

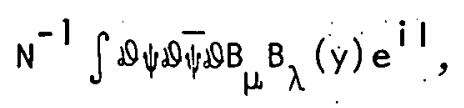

we obtain, after differentiation with respect to $w_{\mu}(x)$,

$$
\begin{aligned}
\left\langle T^{*}\left(\left\{\left[g_{\mu \nu}\left(\partial^{\rho} \partial_{\rho}+M^{2}\right)-\partial_{\mu} \partial_{\nu}\right] B^{\nu}(x)-g \bar{\psi}(x) \gamma_{\mu} \psi(x)\right\} B_{\lambda}(y)\right)\right\rangle_{O} \\
\quad=i g_{\mu \lambda} \delta^{4}(x-y) .
\end{aligned}
$$

An immediate consequence of (5.13) is the equation of motion of $B_{\mu}(x)$

$$
\left[g_{\mu \nu}\left(\partial^{\rho} \partial_{\rho}+M^{2}\right)-\partial_{\mu} \partial_{\nu}\right] B^{\nu}(x)-g \bar{\psi}(x) \gamma_{\mu} \psi(x)=0
$$


We differentiate (5.13) to obtain

$$
\left\langle T^{*}\left(\left\{M^{2} \partial^{\mu} B_{\mu}(x)-g \delta^{\mu}\left(\bar{\psi}(x) \gamma_{\mu} \psi(x)\right)\right\} B_{\lambda}(y)\right\rangle_{0}=i \partial_{\lambda} \delta^{4}(x-y) .\right.
$$

The second term in the left-hand side vanishes as can be proved easily by making a gauge transformation of the fermion fields. It gives the conservation of the current $\bar{\psi}(x) \gamma_{\mu} \psi(x)$ and the vanishing of the equaltime commutator $\left[\bar{\psi}(x) \gamma_{0} \psi(x), B_{\lambda}(y)\right]_{x_{0}}=y_{0} \cdot$ Equation (5.15) then becomes

$$
\left\langle T^{*}\left(\partial_{B_{\mu}}^{\mu_{B}}(x) B_{\lambda}(y)\right)\right\rangle=\frac{i}{M^{2}} \partial_{\lambda} \delta^{4}(x-y)
$$

which gives

$$
\partial_{B_{\mu}}^{\mu_{i}}(x)=0
$$

which is the subsidiary condition; and for $\lambda=k, k=1,2,3$

$$
\left[B_{0}(x), B_{k}(y)\right]_{x_{0}}=y_{0}=\frac{i}{M^{2}} \partial_{k} \delta(x-y) .
$$

Two expressions are obtained from (5.13) by the use of Eq. (5.14) for $\mu=\ell=1,2,3$ and $\lambda=k$,

$$
\begin{aligned}
& \delta\left(x_{0}-y_{0}\right)\left[\partial_{o} B_{\ell}(x)-\partial_{\ell} B_{0}(x), B_{k}(y)\right]+\partial_{0}\left\{\delta\left(x_{0}-y_{o}\right)\left[B_{\ell}(x), B_{k}(y)\right]\right\} \\
& =i g_{\ell k} \delta^{4}(x-y) ; \\
& \quad \therefore \\
& \delta\left(x_{0}-y_{0}\right)\left[\vec{\nabla} \cdot \vec{B}(x), B_{k}(y)\right]=0 .
\end{aligned}
$$

With the assumption that $\left[B_{\ell}(x), B_{k}(y)\right]_{x_{0}}=y_{0}$ : contains $\delta$-function and 
and its finite derivatives, one can see that the above equation is equivalent to

$$
\left[B_{\ell}(x), B_{k}(y)\right]=0 \quad(\ell, k=1,2,3)
$$

which must hold if $B_{\ell}, \ell=1,2,3$, are independent field variables. Substituting (5.20) into (5.18), we obtain

$$
\left[\partial_{o} B_{\ell}(x)-\partial_{\ell} B_{o}(x), B_{k}(y)\right]_{x_{0}}=y_{o}=i g_{\ell k} \delta^{3}(x-y) .
$$

\section{CONCLUSION}

The derivation of general conservation laws is formulated in terms of a local variational method, in which the improved energy-momentum tensor of Callen-Coleman-Jackiw ${ }^{9}$ is derived. Applying the same transformations of the field variables to the functional integration as changes of integration variables, we derive $W-T$ identities involving the energymomentum tensor, its trace, and the SU(3) X SU(3) currents. The framework of the derivation is a vector gluon model containing spin $\frac{1}{2}$ quark fields and a massive unitary singlet vector meson. Using the Feynman rules for the energy-momentum tensor described in Appendix $C$, we have checked that the $W-T$ identities and the trace relations derived in Sec. IV are satisfied naively (to the lowest (zeroth) order of the spinor-gluon coupling constant). Undoubtly, some of the $W-T$ identities and trace identities cannot be satisfied in the explicit calculation when surface terms due to the shift of origin of the integration variables are taken into account. This is analogous with the well-known triangle ${ }^{20}$ and the general anomalies of $W-T$ identities involving currents. ${ }^{21}$ Brown et al., ${ }^{21}$ 
have obtained two sets of miminal solutions for the W-T identity anomalies of SU(3) $\times$ SU(3) currents; hopefully, by requiring a minimal set of solutions for the W-T identities involving energy-momentum tensor, a unique set of the solutions of Brown et al. will emerge. This can be readily investigated by means of the regularization scheme outlined in Sec. 111 and Appendix A. Work on this is in progress.

We also demonstrated that starting from a W-T identity, one can derive the corresponding conservation laws and appropriate equaltime commutation relations. This leads us to the conclusion that the functional integration approach is completely equivalent to the conventional approach.

The virtue of the functional integration approach lies in the following facts: (1) It renders the derivation of $W-T$ identities straightforward. Information of the equal time commutation relations which is indispensable in the conventional approach is unnecessary. The variations of field variables and the corresponding $W-T$, identities discussed in Sec. $I I I$ and IV have covered most of the interesting cases. Other W-T identities can be derived easily once the corresponding variations of the field variables are found. (2) The functional integration defines covariant $\mathrm{T}^{*}$-products and leads to consistent Feynman rules. As an illustration, let us consider. the energy-mementum tensor of a free fermion theory. One may write

$$
\theta_{\mu \nu}(x)=\frac{i}{4}\left[\bar{\psi}(x) \gamma_{\mu} \partial_{\nu} \psi(x)-\partial_{\nu} \bar{\psi}(x) \gamma_{\mu} \psi(x)+\mu \leftrightarrow \nu\right]
$$

by dropping the $-g_{\mu \nu}{ }_{\nu}$ term, since the equations of motion implies that $\mathcal{L}=0$. Then the Feynman rule for the $\theta_{\mu \nu}-\psi-\bar{\psi}$ vertex becomes 


$$
\frac{1}{4}\left[\gamma_{\mu}(p+q)_{\nu}+\gamma_{\nu}(p+q)_{\mu}\right]
$$

where $p$ and $q$ are respectively the momenta of the outgoing and incoming fermions, instead of the rule derived in Appendix C, Eq. (C.1). Obviously (6.2) does not satisfy $(4.25)$ and $(4.26)$. Nevertheless, the W-T identities and trace relations are also changed due to the omission of the term, $-g_{\mu \nu} \mathcal{L}$. The changes can be easily arrived by means of the following identity which holds for a free fermion Lagrangian

$$
\left\langle T^{*}\left(\mathcal{L}(x) \psi\left(y_{1}\right) \cdots \bar{\psi}\left(y_{n}\right)\right)\right\rangle_{0}=\frac{1}{2} \sum_{l=1}^{n} \delta\left(y_{\ell}-x\right)\left\langle T^{*}\left(\psi\left(y_{1}\right) \cdots \bar{\psi}\left(y_{n}\right)\right)\right\rangle_{0} \text {. }
$$

This follows from the field transformation

$$
\psi(x) \rightarrow(1+\omega(x)) \psi(x), \bar{\psi}(x) \rightarrow(1+\omega(x)) \bar{\psi}(x)
$$

and an analysis of the Jacobian of the transformation similar to that described in Appendix B. The right-hand sides of Eqs. (4.24) and (4.25), for examples, are adding, respectively, with the terms

$$
-\frac{1}{2} \frac{\partial}{\partial x p}-\left[\delta^{4}(y-x)+\delta^{4}(z-x)\right]\left\langle T^{*}\left(\psi_{\alpha}(y) \bar{\psi}_{B}(z)\right)\right\rangle_{0}
$$

and

$$
-2\left[\delta^{4}(y-x)+\delta^{4}(z-x)\right]\left\langle T^{*}\left(\psi_{\alpha}(y) \bar{\psi}_{\beta}(z)\right)\right\rangle_{0} \cdot
$$

By these modifications, (4.25) and (4.26) are satisfied indeed with the Feynman rule $(6.2)$. 
VII. ACKNOWLEDGMENTS

One of us (B. L. Y.) wishes to thank Professor R. Jackiw for illuminating discussions of the method of functional integration. He also wishes to thank Professor S. Treiman for the hospitality extended to him at the National Accelerator Laboratory during a summer visit in 1972 when this work began. In addition he would like to thank Frofessor S. Gasiorowicz and Professor D. A. Geffen for their hospitality during several visits to the University of Minnesota. 
APPENDIX A

\section{DER IVATION OF GENERATING FUNCTION}

In this appendix, we derive the generating functional for the Lagrangian (3.1). The generating functional is defined as

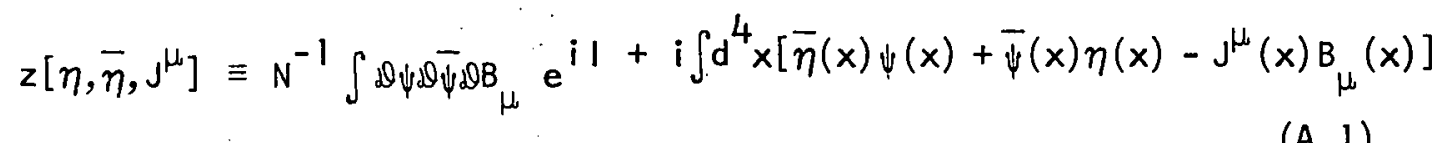

where $N$ is given in Eq. (3.6) and $\eta, \bar{n}, \psi$ and $\bar{\psi}$ are anti-commuting C-numbers, i.e.

$$
\{\eta, \bar{\eta}\}=\{\eta, \psi\}=\{\eta, \bar{\psi}\}=\cdots \cdot=0
$$

We rewrite the term in the exponent,

$$
\begin{aligned}
e^{i I+} & i \int d^{4} x\left[\bar{\eta}(x) \psi(x)+\bar{\psi}(x) \eta(x)-J^{\mu}(x) B_{\mu}(x)\right] \\
& =e^{i \int d^{4} x\left[\bar{\psi}(x) D\left(x, \frac{\delta}{\delta J \mu}\right) \psi(x)\right]+i \int d^{4} x[\bar{\psi}(x) \eta(x)+\bar{\eta}(x) \psi(x)]} \\
& \cdot e^{i \int d^{4} x \frac{1}{2} B^{\mu}(x) K_{\mu \nu}(x) B^{\nu}(x)-i \int d^{4} x J^{\mu}(x) B_{\mu}(x)}
\end{aligned}
$$

where

$$
\begin{aligned}
& D\left(x, \frac{\delta}{\delta J^{\mu}}\right)=i \gamma^{\mu} \partial_{\mu}-m-i g \gamma^{\mu} \frac{\delta}{\delta J^{\mu}(x)} \\
& k_{\mu \nu}(x)=g_{\mu \nu}\left(\partial^{\lambda} \partial_{\lambda}+M^{2}\right)-\partial_{\mu} \partial_{\nu} .
\end{aligned}
$$

Partial integration is used to obtain (A.2). Let us change the variables of integration 


$$
\begin{aligned}
& B_{\mu}(x) \rightarrow B_{\mu}(x)+\int_{\mu \nu}(x, y) J^{\nu}(y) d^{4} y \\
& \psi(x) \rightarrow \psi(x)-\int S_{F}\left(x, y ; \frac{\delta}{\delta J^{\mu}}\right) \eta(y) d^{4} y \\
& \bar{\psi}(x) \rightarrow \bar{\psi}(x)-\int \bar{\eta}(y) S_{F}\left(y, x ; \frac{\delta}{\delta J^{\mu}}\right) d^{4} y
\end{aligned}
$$

with

$$
\begin{aligned}
& g^{\nu \nu^{\prime}}{ }_{\mu \nu \nu}(x) D_{\nu^{\prime} \lambda}(x, y)=g_{\mu \lambda} \delta^{4}(x-y) \\
& D\left(x, \frac{\delta}{\delta J^{\mu}(x)}\right) S_{F}\left(x, y ; \frac{\delta}{\delta J^{\mu}}\right)=\delta^{4}(x-y) \therefore
\end{aligned}
$$

The solutions of $(A .5)$ are

$$
\begin{aligned}
& D_{\nu \lambda}(x, y)=\left(\frac{1}{2 \pi}\right)^{4} \int d^{4} x \frac{\left(k_{\nu}{ }^{k} / M^{2}-g_{\nu \lambda}\right)}{k^{2}-M^{2}+i \epsilon} e^{-i k(x-y)} \\
& S_{F}\left(x, y ; \frac{\delta}{\delta J^{\mu}}\right)=S_{F}(x, y)-\int d z S_{F}(x, z)\left[-i g \gamma^{\mu} \frac{\delta}{\delta J^{\mu}(z)}\right] S_{F}\left(z, y ; \frac{\delta}{\delta J^{\mu}}\right) \\
& S_{F}(x, y)=\left(\frac{1}{2 \pi}\right)^{4} \int d^{4} p \frac{e^{-i p(x-y)}}{b-m+i \epsilon}
\end{aligned}
$$

Substituting (A.4) into (A.1) and (A.2), we obtain

$$
\begin{aligned}
& z\left[\eta, \bar{n}, J^{\mu}\right]=N^{-1} \int 2 \phi \psi \nabla \psi \partial B B_{\mu} e^{+. i \int d^{4} x\left[\bar{\psi}(x) D\left(x, \delta / \delta J^{\mu}(x)\right) \psi(x)+\frac{1}{2} B^{\mu}(x) k_{\mu \nu}(x) B^{\nu}(x)\right]} \\
& e^{-I \int \vec{\eta}(x) s_{F}\left(x, y ; \delta / \delta J^{\mu}\right) \eta(y) d x d y} e^{-\frac{i}{2} \int d x d y J^{\mu}(x) \cdot D_{\mu \nu}(x, y) J^{\nu}(y)} .
\end{aligned}
$$


To evaluate $N$, we write

$$
N=\lim _{j^{\mu} \rightarrow 0} \int d \psi \Delta \bar{\psi} d B_{\mu} e^{i I-i \int d^{4} x j^{\mu}(x) B_{\mu}(x)}
$$

Repeat the same argument, we obtain

$$
\begin{gathered}
N=\lim _{j^{\mu} \rightarrow 0} \int D \psi \Delta D D_{L B} B_{\mu} e^{i \int d^{4} x\left[\bar{\psi}(x) D\left(x, \delta / \delta j^{\mu}\right) \psi(x)+\frac{1}{2} B^{\mu}(x) K_{\mu \nu}(x) B^{\nu}(x)\right]} \\
. e^{-\frac{i}{2} \int d x d y j^{\mu}(x) D_{\mu \nu}(x, y) j^{\nu}(y)}
\end{gathered}
$$

We see from Eqs. (A.7) and (A.9) that the vector meson field can be divided out from the numerator and the denominator of $z\left[\eta, \bar{\eta}, \mathrm{J}^{\mathrm{H}}\right]$. It remains to evaluate the following integral

$$
z\left[\zeta^{\mu}\right]=\int D \psi d \nabla \bar{\psi} e^{i \int d^{4} x \bar{\psi}(x) D\left(x, \zeta^{\mu}\right) \psi(x)}
$$

We shall follow Schwinger's approach. 22

$$
\begin{aligned}
& \frac{\delta \ln z\left[\zeta^{\mu}\right]}{\delta \zeta^{\mu}(x)}=z\left[\zeta^{\mu}\right]^{-1} \int 0 \psi d \bar{\psi} \bar{\psi}(x) g \gamma_{\mu} \psi(x) e^{i \int d^{4} y \bar{\psi}(y) D\left(y, \zeta^{\mu}\right) \psi(y)} \\
& =-i g \operatorname{Tr}\left[\gamma_{\mu} S_{F}\left(x, x ; \zeta^{\mu}\right)\right] .
\end{aligned}
$$

Then

$$
\delta \ln z\left[\zeta^{\mu}\right]=-\operatorname{ig} \int d^{4} x \delta \zeta^{-}(x) \operatorname{Tr}\left[\gamma_{\mu} S_{F}\left(x, x ; \zeta^{\mu}\right)\right]
$$


Symbolically the solution of $S_{F}\left(x, y ; \zeta^{\mu}\right)($ see $(A .6 b))$ is

$$
S_{F}\left(\zeta^{\mu}\right)=\left(1-i g S_{F} \gamma_{\mu} \zeta^{\mu}\right)^{-1} S_{F} .
$$

We obtain

$$
\delta \ln z\left[\zeta^{\mu}\right]=\operatorname{Tr}\left[\delta\left(1-i g S_{F} \gamma_{\mu} \zeta^{\mu}\right)\left(1-i g S_{F} \gamma_{\mu} \zeta^{\mu}\right)^{-1}\right]
$$

where the trace is taken with respect to the spinor indices as well as the infinite space-time coordinates. The solution is

$$
z\left[\zeta^{\mu}\right]=\operatorname{det}\left(1-i g S_{F} \gamma_{\mu} \zeta^{\mu}\right)
$$

We obtain finally

$$
\begin{gathered}
Z\left[n, \bar{\eta}, J^{\mu}\right]=\bar{N}^{-1} \operatorname{det}\left(1-i g S_{F} \gamma^{\mu} \frac{\delta}{\delta J^{\mu}}\right) e^{-i \int d x d y \bar{\eta}(x) S_{F}\left(x, y ; \frac{\delta}{\delta J^{\mu}}\right) \eta(y)} \\
e^{-\frac{i}{2} \int d x d y J^{\mu}(x) D_{\mu \nu}(x, y) J^{\nu}(y)}
\end{gathered}
$$

where

$$
\bar{N}=\lim _{j^{\mu} \rightarrow 0} \operatorname{det}\left(1-i g S_{F} \gamma^{\mu} \frac{\delta}{\delta j^{\mu}}\right) e^{-\frac{i}{2} \int d x d y j^{\mu}(x) D_{\mu \nu}(x, y) j^{\nu}(y)}
$$

is the vacuum-vacuum amplitude ${ }^{23}$ and (A.16) generates the proper amplitudes with the vacuum-vacuum amplitude being divided out.

It is sometimes more convenient to use a different form for the generating function 
42

$$
\begin{aligned}
& e^{i 1+i \int d^{4} x\left[\bar{\eta}(x) \psi(x)+\bar{\psi}(x) \eta(x)-J^{\mu}(x) B(x)\right]} \\
& =e^{\frac{i}{2} \int d^{4} x B^{\mu}(x) K_{\mu \nu}(x) B^{\nu}(x)-i \int d^{4} x\left(J^{\mu}(x)+\xi^{L}(x)\right) B_{\mu}(x)} \\
& \quad \cdot e^{i \int d^{4} x \bar{\psi}(x) D(x) \psi(x)+i \int d^{4} x[\bar{\eta}(x) \psi(x)+\bar{\psi}(x) \eta(x)]}
\end{aligned}
$$

where

$$
\begin{aligned}
\xi^{\mu}(x) & =g \frac{\delta}{\delta \eta(x)} \gamma^{\mu} \frac{\delta}{\delta \bar{\eta}(x)} \\
D(x) & =i \gamma^{\mu} \partial_{\mu}-m .
\end{aligned}
$$

By the change of the integration variables

$$
\begin{aligned}
& B_{\mu}(x) \rightarrow B_{\mu}(x)+\int D_{\mu \nu}(x, y)\left(J^{\nu}(y)+\xi^{\nu}(y)\right) d^{4} \dot{y} \\
& \psi(x) \rightarrow \psi(x)-\int S_{F}(x, y) \eta(y) d^{4} y \\
& \bar{\psi}(x) \rightarrow \bar{\psi}(x)-\int \bar{\eta}(y) S_{F}(x, y) d^{4} y
\end{aligned}
$$

the generating functional becomes

$$
\begin{aligned}
& Z\left[\eta, \bar{\eta}, J^{\mu}\right]=N^{-1} \int d \psi D Q \bar{B}_{\mu} e^{i \int d^{4} x\left[\bar{\psi}(x) D(x) \psi(x)+\frac{1}{2} B^{\mu}(x) K_{\mu \nu}(x) B \nu(x)\right]} \\
& \therefore e^{-\frac{i}{2} \int d^{4} x d^{4} y\left(J^{\mu}(x)+\xi^{\mu}(x)\right) D_{\mu \nu}(x, y)\left(J^{\nu}(y)+\xi^{\nu}(y)\right)} . \\
& \text { - } e^{-i \int d^{4} x d^{4} y \bar{\eta}(x) s_{F}(x, y) \eta(y)} \text {. }
\end{aligned}
$$


Similarly, we can write

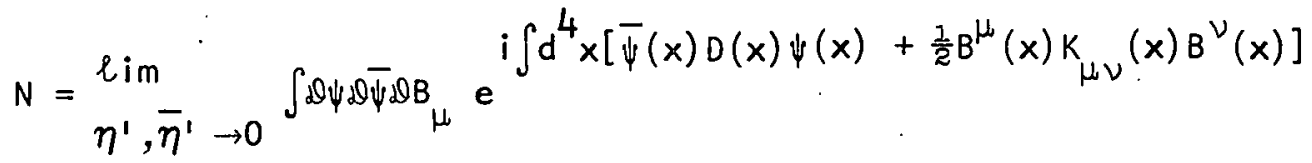

$$
\begin{aligned}
& \text { - } e^{-\frac{i}{2} \int d^{4} x d^{4} y \xi^{\prime \mu}(x) D_{\mu \nu}(x, y) \xi^{\prime \nu}(y)} \\
& \text { - } e^{-i \int d^{4} x d^{4} y \eta^{-1}(x) S_{F}(x, y) \eta^{\prime}(y)}
\end{aligned}
$$

and

$$
\xi^{\prime \mu}(x)=g \frac{\delta}{\delta \eta^{\prime}(x)} \gamma^{\mu} \frac{\delta}{\delta \bar{\eta}^{\prime}(x)} .
$$

Finally, we obtain

$$
\begin{aligned}
z\left[\eta, \bar{\eta}, J^{\mu}\right] & =\bar{N}^{-1} \cdot e^{-\frac{i}{2} \int d^{4} x d^{4} y\left(J^{\mu}(x)+\xi^{\mu}(x)\right) D_{\mu \nu}(x, y)\left(J^{\nu}(y)+\xi^{\nu}(y)\right)} \\
& \cdot e^{-i \int d^{4} x d^{4} y \bar{\eta}(x) S_{F}(x, y) \eta(y)}
\end{aligned}
$$

where

$$
\bar{N}^{\prime}=\lim _{\eta^{\prime}, \bar{\eta}^{\prime} \rightarrow 0} e^{-\frac{i}{2} \int d^{4} x d^{4} y \xi^{\prime \mu}(x) D_{\mu \nu}(x, y) \xi^{\prime} \nu(y)} e^{-i \int d^{4} x d^{4} \cdot y \bar{\eta}^{\prime}(x) S_{F}(x, y) \eta^{\prime}(y)}
$$

Another expression for the generating functional which is more convenient when the interaction is more complicated than we have specified 
in Eq. (3.1) and is suitable for the discussion of regularization can be obtained by rewriting $(A .2)$ or $(A .18)$ in the form

$$
\begin{aligned}
& e^{g \int d^{4} x} \frac{\delta}{\delta \prod(x)} \gamma^{\mu} \frac{\delta}{\delta \bar{\eta}(x)} \frac{\delta}{\delta J^{\mu}(x)} \\
& \quad e^{i \int d^{4} x\left[\bar{\psi}(x) D(x) \psi(x)+\frac{1}{2} B^{\mu}(x) K_{\mu \nu}(x) B^{\nu}(x)\right]} \\
& \quad e^{i \int d^{4} x\left[\bar{\eta}(x) \psi(x)+\bar{\psi}(x) \eta(x)-J^{\mu}(x) B_{\mu}(x)\right]} .
\end{aligned}
$$

The generating functional becomes

$$
\begin{aligned}
z\left[\eta, \bar{\eta} \cdot J^{\mu}\right]= & \left(\bar{N}^{\prime \prime}\right)^{-1} e^{g \int d^{4} x \frac{\delta}{\delta \eta !(x)} \gamma^{\mu} \frac{\delta}{\delta \bar{\eta}(x)} \frac{\delta}{\delta J^{\mu}(x)}} \\
& -i \int d^{4} x d^{4} y\left[\bar{\eta}(x) D(x, y) \eta(y)+\frac{3}{2} J^{\mu}(x) D_{\mu \nu}(x, y) J^{\nu}(y)\right] .
\end{aligned}
$$

To evaluate the regularized generating functional, we apply the following transformations to Eq. (3.18) with the help of Eq. (A.26)

$$
\begin{aligned}
& \psi_{j}(x) \rightarrow \psi_{j}(x)-\int d^{4} x S_{F}^{j}(x, y) \eta_{j}(y) \\
& \bar{\psi}_{j}(x)-\bar{\psi}_{j}(x)-\int d^{4} x \eta_{j}(y) S_{F}^{j}(y, x) \\
& B_{\mu}(x) \rightarrow B_{\mu}(x)+\int d^{4} y D_{\mu \nu}(x, y) J^{\nu}(y) \\
& B_{\mu}^{R}(x) \rightarrow B_{\mu}^{R}(x)-i \int d^{4} y D_{\mu \nu}^{R}(x, y) J^{\prime \nu}(y) .
\end{aligned}
$$


We obtain

$$
\begin{aligned}
& z\left[\eta, \bar{\eta}, J^{\prime \prime}, \Gamma^{\alpha}\right]=N_{R}^{-1} \eta_{l}, \ldots \bar{\eta}_{n}, J^{\prime \mu} \rightarrow 0 \\
& e_{\ell=0}^{n} c_{l} d^{4} x\left\{g \frac{\delta}{\delta \eta_{l}(x)} \gamma^{\mu} \frac{\delta}{\delta \eta_{\ell}(x)}\left[\frac{\delta}{\delta J^{\mu}(x)}+i \frac{\delta}{\delta J^{\prime \mu}(x)}\right]\right. \\
& \left.+i \Gamma^{\alpha}(x) \mathrm{j}_{\alpha}\left(\frac{\delta}{\delta \eta_{\ell}(x)}, \frac{\delta}{\delta \bar{\eta}_{\ell}(x)}, \frac{\delta}{\delta J^{\mu}(x)}, \mathrm{i} \frac{\delta}{\delta J^{\prime \mu}(x)}\right)\right\} \\
& \ldots e^{-i} \sum_{l=0}^{n} \int d x d y \bar{\eta}_{l}(x) S_{F}^{R}(x, y) \eta_{l}(y) \\
& \text { - } e^{-\frac{1}{2} \int d x d y\left[J^{\mu}(x) \cdot D_{\mu \nu}(x, y) J^{\nu}(y)+J^{\prime \mu}(x) D_{\mu \nu}^{R}(x, y) J^{\prime \nu}(y)\right],}
\end{aligned}
$$

where $D_{\mu \nu}^{R}(x, y)$ is similar to $D_{\mu \nu}(x, y)$, Eq. (A. 6a) except that $\mu$ is replaced by $M_{R} ; j_{\alpha}\left(\frac{\delta}{\delta \bar{\eta}_{\ell}(x)}, \cdots\right)$ denotes the expression obtained from the external current $j_{\alpha}(\bar{\psi}(x) \cdots)$ with $\bar{\psi}_{\ell}(x)$; etc. being replaced by $\frac{\delta}{\delta \eta(x)}$, etc. $N_{R}$ is similar to the rest of the expression in (A.28) except that all the external sources go to zero. One can check that in a perturbation expansion of Eq. (A.28), the vector meson propagators are always in the comb ination

$$
D_{\mu \nu}(x, y)-D_{\mu \nu}^{R}(x, y)
$$

and all the fermion loops $g\left(m ; p_{1}, \cdots, p_{j}\right)$ are in the form

$$
\sum_{l=0}^{n} c_{l} g\left(m_{l} ; p_{i}, \cdots, p_{j}\right)
$$


APPENDIX B

JACOBIANS

We shall calculate the Jacobians of the transformations (4.11) and (4.15). Let the field variable be changed by a linear transformation $\varphi^{\prime}(x)=\int k(x, y) \varphi(y) d^{4} y$

If we assume that $k-1$ is infinitesimal, then ${ }^{24}$

$$
J\left(\frac{\delta \varphi^{\prime}}{\delta \varphi}\right)=\left\{\begin{array}{l}
e^{T_{r}} \ln K \approx 1+\operatorname{Tr}(K-1) \text { for Boson field } \\
e^{-T_{r}} \ln K \approx 1-\operatorname{Tr}(K-1) \text { for fermion field }
\end{array}\right.
$$

$K$ is a matrix in the coordinate space as well as the spin space. 
For the Transformation (4.11):

$$
\begin{aligned}
& \left\langle x\left|\left(k_{\psi}-1\right)\right| y\right\rangle=\omega^{\lambda}(x) \frac{\partial}{\partial x^{\lambda}} \delta(x-y)+\frac{1}{8} \partial^{\lambda}{ }_{\omega}^{\rho}(x)\left[\gamma_{\lambda}, \gamma_{\rho}\right] \delta(x-y) \\
& \left\langle x\left|\left(k_{\psi}-1\right)\right| y\right\rangle=\omega^{\lambda}(x) \frac{\partial}{\partial x^{\lambda}} \delta(x-y)-\frac{1}{8} \partial^{\lambda}{ }_{\omega}^{\rho}(x)\left[\gamma_{\lambda}, \gamma_{\rho}\right] \delta(x-y) \\
& \left\langle x\left|\left(k_{B_{\mu}}-1\right)_{\mu \nu}\right| y\right\rangle=g_{\mu \nu} \omega^{\lambda}(x): \frac{\partial}{\partial x^{\lambda}} \delta(x-y)+\partial^{\lambda} \omega^{\rho}(x)\left(g_{\mu \lambda} g_{\nu \rho}-g_{\mu \rho} g_{\nu \lambda}\right) .
\end{aligned}
$$

The second terms in the right-hand sides of. (B.3) do not contribute, their traces with respect to the spin indices vanish. In the momentum representation, the first terms of (B.3) are proportional to

$$
\begin{gathered}
\int d^{4} x d^{4} y e^{i p x} \omega^{\lambda}(x) \frac{\partial}{\partial x^{\lambda}} \cdot \delta(x-y) e^{-i p^{\prime} y} \\
=i p_{\lambda} \int d^{4} x \omega^{\lambda}(x) e^{-i\left(p-p^{\prime}\right) x}
\end{gathered}
$$

which does not contribute to the traces summing over the four-momenta. Therefore, we have $J\left(\delta \varphi^{\prime} / \delta \varphi\right)=1$.

For the transformation (4.15):

Using a discrete label, we write

$$
\left\langle x\left|\left(k_{\varphi}-1\right)\right| y\right\rangle=d_{\varphi}^{\omega(x)} \delta_{x y}
$$

for $\varphi=\psi, \bar{\psi}$, or $B_{\mu}$; where $d_{\psi}=\alpha_{\psi}=3 / 2$ and $d_{B}=1$. 


$$
\begin{aligned}
\operatorname{Tr}\left(K_{\varphi}-1\right) & =c \Sigma_{x} \frac{\left\langle x\left|\left(K_{\varphi}-1\right)\right| x\right\rangle}{\langle x \mid x\rangle} \\
& =c \Sigma_{x} d_{\varphi} \omega(x) \\
& =d \varphi^{C} \lim _{\Delta x \rightarrow 0}\left(\frac{1}{\Delta x}\right)^{4} \int \omega(x) d^{4} x
\end{aligned}
$$

and

$$
=d_{\varphi} c \delta^{4}(x=0) \int d^{4} x w(x)
$$

where $C=4$ for spin 1 and $\frac{1}{2}$ fields and $C=1$ for spin 0 field. In (B.6) we have used the following relations

$$
\lim _{\Delta x \rightarrow 0}\left(\frac{1}{\Delta x}\right)^{4}=\delta^{4}(x=0)
$$

which can be proved by passing to the infinite space-time limit from the box normalization. Hence the Jacobian appearing in Eqs. (4.18) and (4.19) is

$$
\begin{aligned}
J(\omega) & =J_{x}(\omega) J_{\bar{x}}(\omega) J_{B_{\mu}}(\omega) \\
& \simeq 1-T_{r}\left(K_{\psi}-1\right)-T_{r}\left(K_{\bar{\psi}}-1\right)+T_{r}\left(K_{B_{\mu}}-1\right) \\
& =1-8 \delta^{4}(x=0) \int d^{4} x w(x)+0 .\left(\omega_{-}^{2}\right) . .
\end{aligned}
$$

Therefore

$$
\frac{\delta J(\omega)}{\partial \omega(x)}=-8 \delta^{4}(x=0) .
$$

It remains to calculate $\left\langle T^{*}\left(\theta_{\mu}^{\mu}(x)-\theta(x)\right)\right\rangle_{0}$. 
This is needed in deriving the trace identity. From Eqs. (3.2) and (3.3), we write

$$
\begin{aligned}
\left\langle T^{*}\left(\theta_{\mu}^{\mu}(x)-\theta(x)\right)\right\rangle_{0}= & -3\left\langle T^{*}\left(\bar{\psi}(x)\left(i \gamma^{\mu} \partial_{\mu}-m-g \gamma^{\mu} B_{\mu}(x)\right) \psi(x)\right)\right\rangle_{0} \\
& -\left\langle T^{*}\left(\left(k_{\mu \nu}(x) B^{\nu}(x)-g \bar{\psi}(x) \gamma_{\mu} \psi(x)\right) B^{\mu}(x)\right)\right\rangle_{0} .
\end{aligned}
$$

From Eq. (A.16) we write,

$$
\begin{aligned}
& -3\left\langle T^{*}\left(\bar{\psi}(x)\left(i \gamma_{\mu}^{\mu} \partial_{\mu}-m-g \gamma^{\mu} B_{\mu}(x)\right) \psi(x)\right)\right\rangle_{o} \\
& \left.=3 \operatorname{Tr}\left\langle T^{*}\left(i \gamma^{\mu} \partial_{\mu}-m-g \gamma^{\mu} B_{\mu}(x)\right) \psi(x) \cdot \bar{\psi}(x)\right)\right\rangle_{o} \\
& =3 \lim _{y \rightarrow x} \operatorname{Tr}\left\{\left.\left\langle\left(i \gamma^{\mu} \partial_{\mu}-m-g \gamma^{\mu} B_{\mu}(x)\right) \frac{\delta}{\delta \bar{\eta}(x)} \frac{\delta}{\delta \eta(y)} \delta^{Z}\left[\eta, \bar{\eta}, J^{\mu}\right]\right\}\right|_{\eta=\bar{\eta}=J^{\mu}=0} .\right. \\
& =12 i \delta^{4}(x=0) .
\end{aligned}
$$

We use (A.24) to write

$$
\begin{aligned}
& \left\langle T^{*}\left(\left(K_{\mu \nu}(x) B^{\nu}(x)-g \bar{\psi}(x) \gamma_{\mu} \psi(x)\right) B^{\mu}(\lambda)\right)\right\rangle_{0} \\
= & \left.\lim _{y \rightarrow x}\left\{\frac{\delta}{\delta J_{\mu}(y)}\left[K_{\mu \nu}(x) \frac{\delta}{\delta J_{\nu}(x)}-g \frac{\delta}{\delta \eta(x)} \gamma_{\mu} \frac{\delta}{\delta \bar{\eta}(x)}\right] z\left[\eta, \bar{\eta}, J^{\mu}\right]\right\}\right|_{\eta=\bar{\eta}=J^{\mu}=0} \\
= & 4 i \delta^{4}(x=0) .
\end{aligned}
$$


Equations (B.10) and (B. 11) give

$$
\left.\left\langle T * \theta_{\mu}^{\mu}(x)-\theta(x)\right)\right\rangle_{0}=8 i \delta^{4}(x=0) .
$$

The Eq. (4.19) follows immediately from (B.8) and (B.12).

\section{APPENDIX C}

\section{FEYNMAN RULES}

Feynman rules for graphs involving the energy momentum tensor and its trace $^{25}$ are given in Fig. 1 and Fig. 2. In Fig. $2, \Gamma_{\mu \nu}^{(1)}(p, q)$ and $\left[\Gamma_{\mu \nu}^{(2)}(p, q)\right]_{\sigma \tau}$ and respectively

$$
\Gamma_{\mu \nu}^{(1)}(p, q)=\frac{1}{4} \gamma_{\mu}(p+q)_{\nu}+\frac{1}{4} \gamma_{\nu}(p+q)_{\mu}-\frac{1}{2} g_{\mu \nu}(\not p+\not q-2 m)
$$

and

$$
\begin{aligned}
{\left[\Gamma_{\mu \nu}^{(1)}(p, q)\right]_{\sigma \tau}=} & -\left(p_{\mu} q_{\nu}+q_{\mu} p_{\nu}\right) g_{\sigma \tau}-p \cdot q\left(g_{\mu \sigma} g_{\nu \tau}+g_{\mu \tau} g_{\nu \sigma}\right. \\
& +p_{\tau}\left(q_{\mu} g_{\nu \sigma}{ }^{\prime}+q_{\nu} g_{\mu \sigma}\right)+q_{\sigma}\left(p_{\mu} g_{\tau \nu}+p_{\nu} g_{\mu \tau}\right) \\
& +\frac{1}{2}\left(p^{2}+q^{+}\right)\left(g_{\mu \nu}+g_{\nu \tau}+g_{\mu \tau} g_{\nu \sigma}\right) \\
& -\frac{2}{2} p_{\sigma}\left(p_{\mu} g_{\nu \tau}+p_{\nu} g_{\mu \tau}\right)-\frac{1}{2} q_{\tau}\left(q_{\mu} g_{\nu \sigma}+q_{\nu} g_{\mu \sigma}\right) \\
& +g_{\mu \nu}\left(p \cdot q g_{\sigma \tau}-p_{\tau} q_{\sigma}\right)-M^{2} g_{\mu \nu} g_{\sigma \tau}
\end{aligned}
$$

It can be checked easily to the lowest order in $g$ that the Feynman rules (i) and (iv) listed in Fig. 2 satisfy the W-T identity (4.24) 
and trace relation (4.25). Rules ( $i i)$ and (v) satisfy the similar identities for the energy-momentum tensor-vector meson vertex. 


\section{REFERENCES}

1. R. P. Feynman, Rev. Mod. Phys. 20, 367 (1948).

2. R. P. Feynman, Phys. Rev. 80, 440 (1950); Phys. Rev. 76, 749, 769 (1949). See also R. P. Feynman and A. R. Hịbs, "Quantum Mechanics and Path Integrals," McGraw-Hill, 1965.

3. The list of the earlier work on the various aspects of the functional integration approach to field-theory is extensive. The following is at best an incomplete list. We apologize for many work being omitted. V. K. Synmansik; Z. Naturforschung 9A, 809 (1954); S. F. Edwards and R. E. Peierls, Proc. Roy. Soc. London, A224, 24 (1954) P. T. Matthews and A. Salam, Nuovo Cimento 2, 120 (1955); J. C. Polkinghorne, Proc. Roy. Soc. London, A 230, 272 (1955).

4. Extensive treatment and review of the functional integration approach to quantum field-theory can be found in the following work: Yu V. Novozhilov and A. V. Tulub, Uspehki Fizicheskikh, Nauk LXI, 53 (1957); English translation in "Advances in. Physical Sciences,". also "The Method of Functionals in the Quantum Theory of Fields," Gordon and Beach, New York, 1961. Jan Rzewuski, Field Theory, Vol. 2 Functional Formulation of S-Matrix theory, 11 iffe Books Ltd., London, 1969. A brief introduction to the functional integration method can be found in D. Lurie, Particles and Fields, Interscience Publishers, 1968. Chapter 10. For the most recent work, see the elegant treatment by V. N. Popor and L. D. Faddeev, Purbation Theory for Gauge-Invariant Fields, ed. by D. Gordon and B. W. Lee, unpublished, NAL-Thy-57, May, 1972. For a most recent extensive review, see E. S. Abers and B. W. Lee, Lectures on Gauge Theory (State Univ. of New York, Stony Brook, N. Y.). 
5. See, for examples, L. D. Faddeev and V. N. Popov, Ref. 4 and Phys. Lett. 25B, 29 (1967); E. S. Fradkin and 1. V. Tyutin, Phys. Letters, 30B, 562 (1969); Phys. Rev. D2, 2841 (1970)。 G.'t Hooft, B33, 173 (1971), ibid B35, 167 (1971); G.'t Hooft and M. Veltman, Nucl. Phys. B44, 189 (1972); B. W. Lee and J. Zinn-Justin, Phys. Rev. D5, 3121 (1972). See for a general summary, J. C. Taylor, An Introduction to Gauge-Fields and Renormalizable Theories of Charged Vector Mesons. (Rutherford High Energy Laboratory, unpublished) and Abers and Lee, Ref. 4.

6. C. S. Hsue, B. Sakita, and M. A. Virasoro, Phys. Rev. D2, 2857 (1970); J.-L. Gervais and B. Sakita, Phys. Rev. D4, 2291 (1971); Nucl. Phys. B34, 477 (1971); Nucl. Phys. 834, 632 (1971).

7. D. J. Gross and R. Jackiw, Nucl. Phys. B 14, 269. (1969).

8. T. Banks, Phys. Rev. D5, 2959 (1972).

9. C. G. Callan, Jr., S. Coleman, and R. Jackiw, Ann. Phys. (New York) 59, $42(1970)$.

10. M. Gell-Mann and M. Lévy, Nuovo Cimento 16, $705^{\prime}$ (1960).

11. For a discussion of the local variation, see, for example, K. Nishijima, Fields and Particles, Field Theory and Dispersion Relations, W. A. Benjamin Inc. New York, 1969, pp. 14-15.

12. $\Lambda_{\mu \nu}=0$ is a consequence of Poincare invariance. See the discussion in R. Jackiw, Dilatation Symmetry and Light-Cone Commutators, lectures delivered at Univerșity of Turin and DESY Summer School, Summer 1972, Chapter 2.

13. See, for example J. M. Jauch and F. Rohrlich, The Theory of Photons and Electrons, Addison-Wesley, Reading, Mass., 1955; pp. 21-22. 
14. For a general discussion of this, see, R. Jackiw, Lectures on

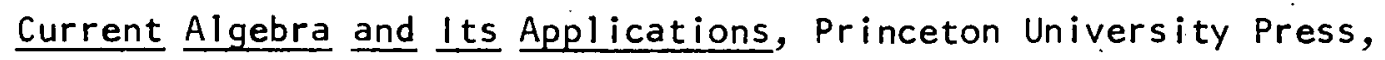
Princeton, 1972, Chapter 7.

15. S. Gasiorowicz and D. A. Geffen, Rev. Mod. Phys. 41, 531 (1969).

16. K. Nishijima, Prog. Theor. Phys. 5, 813 (1950). Z. Koba, ibid. 5, $696(1950)$.

17. W. Pauli and F. Villars, Rev. Mod. Phys. 21, 434 (1949); S. N. Gupta, Proc. Phys. Soc. (London) A66, 129 (1953). See, also B. W. Lee, Phys. Rev. D5, 823 (1972).

18. R. F. Dashen and S. Y. Lee, Phys. Rev. 187, 2017 (1969).

19. The general procedure for deriving canonical commutation relations of fields is given by K. Nishijima, see Ref. 11 .

20. S. Adler, Phys. Rev. 177, 2426. (1969); j. S. Bell and R. Jackiw, Nuovo Cimento 60A, 47 (1969); J.'Schwinger, Phys. Rev. 82, 664 (1951).

21. W. A. Bardeen, Phys. Rev. 184, 1848 (1969); 1. S. Gerstein and R. Jackiw, Phys. Rev. 181, 1955 (1969); R. W. Brown, C. C. Shih, and Bing-lin Young, Phys. Rev. 186, 1491 (1969).

22. J. Schwinger, Phys. Rev. 93, 615 (1954).

23. See the discussion in Lurie, cited in Ref. 4, pp. 468-470.

24. See, for examples, V. K. Synmansik Ref. 3; and Jan Rzewuski, Ref. 4, pp. 262-266 and 276-279.

25. The Feynman rule for the $\theta_{\mu \nu}-\psi-\bar{\psi}$ vertex for a free fermion theory has been obtained by $R$. Jackiw (private communication) prior to the present investigation. 


\section{FIGURE CAPTIONS}

Fig. 1. A wavy line represents the propagator of the vector meson and a straight represents a spinor.

Fig. 2. Feynman rules for vertices involving the energy-momentum tensor and its trace. For the meanings of the various symbols see Fig. 1. $\Gamma_{\mu \nu}^{(1)}(p, q)$ and $\left[\Gamma_{\mu \nu}^{(2)}(p, q)\right]_{\mu \nu}$ are given respectively by Eqs. (C.I) and (C.2). 
$\frown \sim_{k}\left(k_{\mu} k_{\nu} / M^{2}-g_{\mu \nu}\right) /\left(k^{2}-M^{2}\right)$

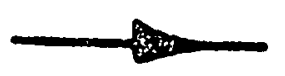

$i /(p-m)$

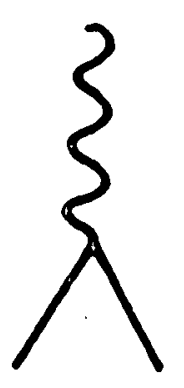

$-\operatorname{igY}_{\mu}$

an external line representing energy-momentum tensor $\theta_{\mu \nu}$

-

trace of energy-momentum tensor

Fig. 1. A wavy line represents the propagator of the vector meson and a straight represents a spinor. 
(i)

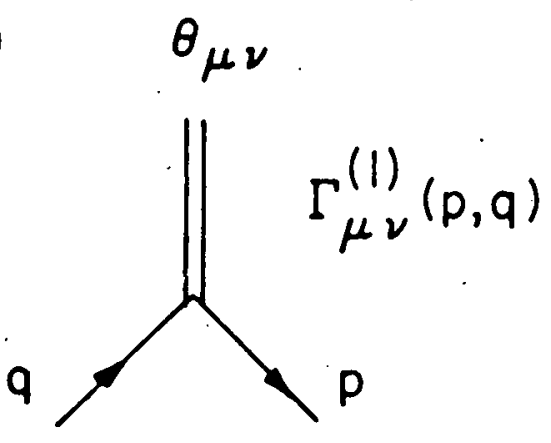

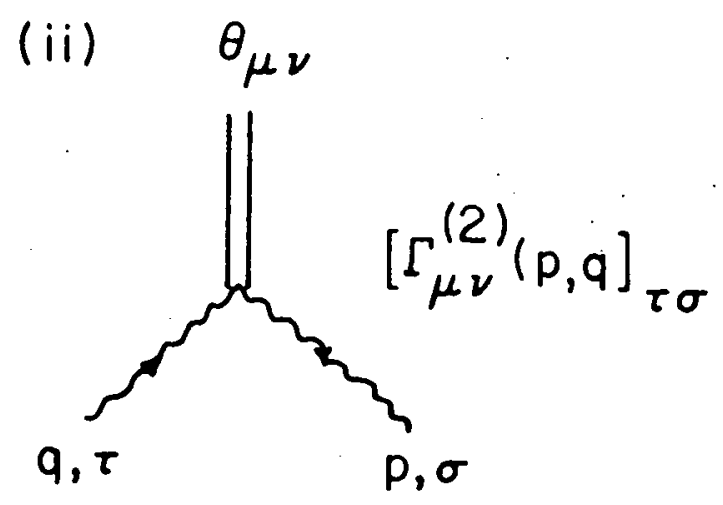

(iv)

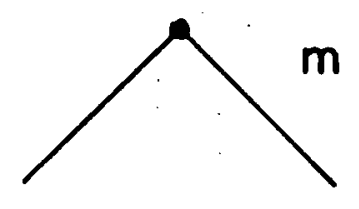

(v).

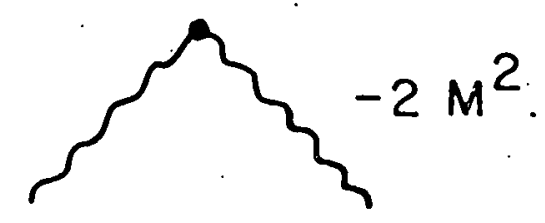

Fig. 2. Feynman rules for vertices involving the energy-momentum tensor and its trace. For the meanings of the various symbols see Fig. 1. $\Gamma_{\mu \nu}^{(1)}(p, q)$ and $\left[\Gamma_{\mu \nu}^{(2)}(p, q)\right]_{\mu \nu}$ are given respectively by Eqs. (C.1) and (C.2). 